\title{
Rank one groups and division pairs
}

\author{
Ottmar Loos
}

\begin{abstract}
Rank one groups were introduced by F. G. Timmesfeld as the building blocks of Lie type groups. Division pairs are algebraic objects categorically equivalent to Moufang sets. We define a functor $\Delta$ from rank one groups to division pairs and prove that $\Delta$ has a left adjoint $\Sigma$, given by a Steinberg type construction. We also extend the theory of the quasi-inverse and the Bergmann operators, well known from Jordan pairs, to this setting. As an application, we show that identities proved by T. De Medts and Y. Segev for Moufang sets hold in arbitrary rank one groups.
\end{abstract}

\section{Introduction}

A rank one group is a group $G$ together with a pair $\left(U^{+}, U^{-}\right)$of subgroups which generate $G$, have trivial intersection and satisfy the following condition: for every $\sigma \in\{+,-\}$ and $x \in \dot{U}^{\sigma}:=U^{\sigma}-\{1\}$ there exists $y \in U^{-\sigma}$ such that

$$
x U^{-\sigma} x^{-1}=y U^{\sigma} y^{-1} .
$$

Rank one groups (with a slightly more restrictive definition) were introduced by F. G. Timmesfeld [12] as the building blocks of Lie type groups. They are essentially equivalent to split saturated Tits systems of rank one, and have close connections to Moufang sets [15, 3].

Division pairs were defined and shown to be categorically equivalent to Moufang sets in [9]. Briefly, a division pair is a pair $V=\left(V^{+}, V^{-}\right)$of groups together with a pair $\left(j_{+}, j_{-}\right)$of bijections $j_{\sigma}$ from the non-trivial elements of $V^{\sigma}$ to those of

Received by the editors in March 2013 - In revised form in December 2013.

Communicated by M. Van den Bergh.

2010 Mathematics Subject Classification : Primary: 17C60, 20E42, Secondary: 17C30.

Key words and phrases : Rank one group, division pair, Moufang set, quasi-inverse, Bergmann operator, identities . 
$V^{-\sigma}$ such that the so-called $R$-operators, defined in a fairly complicated way in terms of the maps $j_{\sigma}$ (see Lemma 1.2), are group homomorphisms.

For a rank one group $\mathscr{G}=\left(G, U^{+}, U^{-}\right)$, Timmesfeld [12] showed that the element $y$ in $(*)$ is uniquely determined by $x$, and that $j_{\sigma}(x):=y$ defines a bijection $j_{\sigma}: \dot{U}^{\sigma} \rightarrow \dot{U}^{-\sigma}$. We show in Proposition 2.7 that the pair of groups $\left(U^{+}, U^{-}\right)$ together with these maps $j_{\sigma}$ is a division pair, denoted $\Delta(\mathscr{G})$; in fact, with an appropriate definition of morphism for rank one groups, $\Delta$ is a functor from rank one groups to division pairs.

Two natural questions arise here:

(i) Does every division pair come from a rank one group?

(ii) To what extent is a rank one group determined by its associated division pair?

We show that the answer to (i) is yes, and the answer to (ii) is almost yes: a rank one group is uniquely determined by its associated division pair up to central extensions.

The more precise formulation is as follows: the functor $\Delta$ has a left adjoint $\Sigma$, a functor from division pairs to rank one groups. For every division pair $V$, the unit of the adjunction is an isomorphism $V \cong \Delta(\Sigma(V))$ while for every rank one group $\mathscr{G}$, the co-unit $\Sigma(\Delta(\mathscr{G})) \rightarrow \mathscr{G}$ is a surjective homomorphism with central kernel (Theorem 3.7).

It may be helpful to compare this result with the strictly analogous situation in the theory of Lie groups. The analogue of $\Delta$ is the Lie algebra of a connected real Lie group while $\Sigma$ corresponds to the construction of the simply connected Lie group for a given Lie algebra. The same situation occurs for compact semisimple Lie groups.

The rank one group $\Sigma(V)$ is obtained by a Steinberg type construction. In more detail, $\Sigma(V)=\left(\hat{G}, \hat{U}^{+}, \hat{U}^{-}\right)$where $\hat{G}$ is the group presented by generators $\hat{\mathrm{e}}_{\sigma}(z), z \in V^{\sigma}, \sigma= \pm$, and the following relations: the $\hat{\mathrm{e}}_{\sigma}: V^{\sigma} \rightarrow \hat{\mathrm{G}}$ are group homomorphisms and

$$
\hat{\mathrm{w}}_{\sigma}(x) \hat{\mathrm{e}}_{-\sigma}(y) \hat{\mathrm{w}}_{\sigma}(x)^{-1}=\hat{\mathrm{e}}_{\sigma}\left(R_{x} y\right)
$$

for all $x \in V^{\sigma}-\{0\}$ and $y \in V^{-\sigma}$, where $\hat{\mathrm{w}}_{\sigma}(x)=\hat{\mathrm{e}}_{-\sigma}\left(-j_{\sigma}(x)\right) \hat{\mathrm{e}}_{\sigma}(x) \hat{\mathrm{e}}_{-\sigma}\left(j_{\sigma}(-x)\right)$. Here the group law in $V^{\sigma}$ is written additively (although these groups need not be abelian) and the $R_{x}$ are the $R$-operators of the division pair $V$. One puts $\hat{U}^{\sigma}=\hat{\mathrm{e}}_{\sigma}\left(V^{\sigma}\right)$. The proof of $V \cong \Delta(\Sigma(V))$ requires $\hat{\mathrm{e}}_{\sigma}: V^{\sigma} \rightarrow \hat{U}^{\sigma}$ to be an isomorphism. It is at this point that the categorical equivalence between division pairs and Moufang sets enters in an essential way.

The construction of the Steinberg group is well known in special cases. If $V^{ \pm}=k$ is a field and $j_{\sigma}(x)=-x^{-1}$ then $R_{x} y=x^{2} y$ and $\hat{G}$ is Steinberg's group $\mathrm{St}_{2}(k)[11, \S 6]$. If $V$ is a Jordan division pair then $\hat{G}$ is the Steinberg group introduced in [8]. In [14, Theorem (2.1)], Timmesfeld defines a Steinberg group for what he calls $K$-structures. These turn out to be special cases of division pairs (cf. 3.2(b)), so his result is contained in ours.

The last section is devoted to identities in rank one groups and division pairs. We also introduce, for division pairs, the notions of quasi-inverse and Bergmann operators, well known from Jordan theory, and develop their basic theory. As an 
application, we show that the identities proved by De Medts and Segev [2] for the so-called $\mu$-operators in the little projective group of a Moufang set hold in fact in an arbitrary rank one group.

Notation. The inner automorphism determined by an element $g$ of a group $G$ is $\operatorname{Int}(g): h \mapsto g h g^{-1}$. The set of non-trivial elements of $G$ is denoted $\dot{G}$, the centre of $G$ is $\mathscr{Z}(G)$, the normalizer of a subset $S$ is $\operatorname{Norm}_{G}(S)$, and the set of subgroups of $G$ is $\operatorname{sbgr}(G)$. We write $((a, b))=a b a^{-1} b^{-1}$ for the group commutator. The index $\sigma$ takes values in $\{+,-\}$ where we regard + and - as abbreviations for +1 and -1 , respectively. The meaning of $-\sigma$ is then the obvious one. The symmetric group of a set $X$ is $\operatorname{Sym}(X)$.

\section{Preliminaries}

In this section, we review the main results about division pairs and Moufang sets from [9].

1.1 Pre-division pairs. A pre-division pair is a pair $V=\left(V^{+}, V^{-}\right)$of groups together with a pair of maps $j_{\sigma}: \dot{V}^{\sigma} \rightarrow \dot{V}^{-\sigma}$ which are inverses of each other:

$$
j_{-\sigma} \circ j_{\sigma}=\operatorname{Id}_{\dot{V}^{\sigma}} \quad(\sigma \in\{+,-\}) .
$$

By abuse of language, we often speak of $V$ as of a pre-division pair, the maps $j_{\sigma}$ being understood from the context. A homomorphism $h=\left(h_{+}, h_{-}\right): V \rightarrow W$ of pre-division pairs is a pair of injective group homomorphisms $h_{\sigma}: V^{\sigma} \rightarrow W^{\sigma}$ such that, for all $\sigma \in\{+,-\}$ and all $x \in \dot{V}^{\sigma}$,

$$
h_{-\sigma}\left(j_{\sigma}(x)\right)=j_{\sigma}\left(h_{\sigma}(x)\right) .
$$

With these definitions, pre-division pairs form a category, denoted pre-div.

Let $V$ be a pre-division pair. We write the group law additively, even though the $V^{\sigma}$ need not be abelian, and denote the group inverse in $V^{\sigma}$ by $i(x)=-x$. For an element $x \in \dot{V}^{\sigma}$, we often write simply $j(x)$ instead $j_{\sigma}(x)$, so that formally $j(j(x))=x$. We also define

$$
{ }^{\vee} x=i(j(x))=-j(x), \quad x^{\vee}=j(i(x))=j(-x) .
$$

Thus both ${ }^{\vee} x$ and $x^{\vee}$ belong to $\dot{V}^{-\sigma}$, the maps $x \mapsto{ }^{\vee} x$ and $x \mapsto x^{\vee}$ are bijections $\dot{V}^{\sigma} \rightarrow \dot{V}^{-\sigma}$ which are inverses of each other:

$$
{ }^{\vee}\left(x^{\vee}\right)=\left({ }^{\vee} x\right)^{\vee}=x,
$$

and we have

$$
(-x)^{\vee}=-{ }^{\vee} x=j(x), \quad{ }^{\vee}(-x)=-x^{\vee}=i j i(x) .
$$

If $h: V \rightarrow V^{\prime}$ is a homomorphism of pre-division pairs, then

$$
h_{-\sigma}\left({ }^{\vee} x\right)={ }^{\vee} h_{\sigma}(x), \quad h_{-\sigma}\left(x^{\vee}\right)=h_{\sigma}(x)^{\vee} .
$$


The opposite of $(V, j)$ is the pre-division pair $(V, j)^{\mathrm{op}}=\left(\left(V^{-}, V^{+}\right),\left(j_{-}, j_{+}\right)\right)$. For a morphism $h=\left(h_{+}, h_{-}\right): V \rightarrow W$ of pre-division pairs, let $h^{\text {op }}=\left(h_{-}, h_{+}\right)$: $V^{\text {op }} \rightarrow W^{\mathrm{op}}$. Then ( $)^{\mathrm{op}}$ is a functor from pre-div to itself whose square is the identity.

1.2 Lemma. Let $V$ be a pre-division pair and $x \in \dot{V}^{\sigma}$.

(a) There exists a unique bijection $R_{x}: V^{-\sigma} \rightarrow V^{\sigma}$, given as follows. If $y=0$ then $R_{x} y=0$. If $x^{\vee}+y=0$ then

$$
R_{x} y=j(x)^{\vee}=j\left({ }^{\vee} x\right) .
$$

If $y \neq 0$ and $x^{\vee}+y \neq 0$ then also $x+j\left(x^{\vee}+y\right) \neq 0$ and ${ }^{\vee} x+j\left(x+j\left(x^{\vee}+y\right)\right) \neq 0$, and

$$
R_{x} y=j\left({ }^{\vee} x+j\left(x+j\left(x^{\vee}+y\right)\right)\right) .
$$

(b) If $h: V \rightarrow W$ is a morphism of pre-division pairs, then the $R$-maps are compatible with $h$ in the sense that

$$
h_{\sigma}\left(R_{x} y\right)=R_{h_{\sigma}(x)} h_{-\sigma}(y) .
$$

Proof. This follows from [9, Lemma 1.5].

1.3 Definition. A division pair is a pre-division pair $V=\left(V^{+}, V^{-}\right)$with the property that, for every $\sigma \in\{+,-\}$ and all $x \in \dot{V}^{\sigma}$, the maps $R_{x}: V^{-\sigma} \rightarrow V^{\sigma}$ are group homomorphisms. We denote the full sub-category of pre-div whose objects are division pairs by div.

A more common but equivalent version of the "Hua identity" (1.2.2) is given in the following lemma.

1.4 Lemma. Let $V$ be a pre-division pair and let $x \in \dot{V}^{\sigma}$.

(a) There exists a well-defined map $\tilde{R}_{x}: V^{-\sigma} \rightarrow V^{\sigma}$, given as follows. If $y=0$ then $\tilde{R}_{x} y=0$. If $y=x^{\vee}$ then

$$
\tilde{R}_{x} y={ }^{\vee \vee} x .
$$

If $0 \neq y \neq x^{\vee}$ then also $x+j(y) \neq 0$ and ${ }^{\vee} x+j(x+j(y)) \neq 0$, and

$$
\tilde{R}_{x} y={ }^{\vee}{ }^{\vee} x+j\left({ }^{\vee} x+j(x+j(y))\right) .
$$

(b) $R_{x}$ is a group homomorphism if and only if $\tilde{R}_{x}$ is, and then $R_{x}=\tilde{R}_{x}$.

Proof. (a) Suppose $0 \neq y \neq x^{\vee}$. Then also $-j(y)={ }^{\vee} y \neq{ }^{\vee}\left(x^{\vee}\right)=x$, so $x+j(y) \neq$ 0 . Assume ${ }^{\vee} x+j(x+j(y))=0$. Then ${ }^{\vee} x=-j(x+j(y))={ }^{\vee}(x+j(y))$ which implies $x=x+j(y)$ and therefore $j(y)=0$, contradiction. Thus $\tilde{R}_{x}$ is well defined.

(b) Suppose $R_{x}$ is a group homomorphism. We show that $\tilde{R}_{x} y=R_{x} y$ for all $y$. If $y=0$ this is clear by definition. Now let $0 \neq y=x^{\vee}$. Then $\tilde{R}_{x} y={ }^{\vee} v$ by (1.4.1) 
while $R_{x} y=-R_{x}(-y)$ (since $R_{x}$ is a group homomorphism) $=-R_{x}\left(-x^{\vee}\right)=$ $-j\left({ }^{\vee} x\right)($ by $(1.2 .1))={ }^{\vee} v$. Finally, let $0 \neq y \neq x^{\vee}$ so that $\tilde{R}_{x} y$ is given by (1.4.2). Put $y^{\prime}=-x^{\vee}+y$. Then $y^{\prime} \neq 0$ and $x^{\vee}+y^{\prime}=y \neq 0$, so by (1.2.2),

$$
R_{x}\left(y^{\prime}\right)=j\left({ }^{\vee} x+j\left(x+j\left(x^{\vee}+y^{\prime}\right)\right)\right)=j\left({ }^{\vee} x+j(x+j(y))\right) .
$$

On the other hand, since $R_{x}$ is a group homomorphism, we have by (1.2.1),

$$
R_{x} y^{\prime}=R_{x}\left(-x^{\vee}+y\right)=j(x)^{\vee}+R_{x} y .
$$

Comparing (1.4.3) and (1.4.4) yields, by (1.4.2),

$$
R_{x} y=-j(x)^{\vee}+j\left({ }^{\vee} x+j(x+j(y))\right)={ }^{\vee}{ }^{\vee} x+j\left({ }^{\vee} x+j(x+j(y))\right)=\tilde{R}_{x} y .
$$

Hence also $\tilde{R}_{x}$ is a group homomorphism.

Conversely, assuming that $\tilde{R}_{x}$ is a group homomorphism, we show that $R_{x}=$ $\tilde{R}_{x}$. For $y=0$ there is again nothing to prove. Now let $y=-x^{\vee}$. Then $R_{x} y=j\left({ }^{\vee} x\right)$ by (1.2.1) while $\tilde{R}_{x} y=-\tilde{R}_{x} x^{\vee}$ (since now $\tilde{R}_{x}$ is a homomorphism) $=-{ }^{\vee} v$ (by $(1.4 .1))=j i j(x)=j\left({ }^{\vee} x\right)$.

Finally, let $y \neq 0$ and $x^{\vee}+y \neq 0$ and put $y^{\prime}=x^{\vee}+y$. Then $y^{\prime} \neq 0$ and $y^{\prime} \neq x^{\vee}$ (since $y \neq 0$ ). Hence by (1.4.2),

$$
\tilde{R}_{x} y^{\prime}={ }^{\vee}{ }^{\vee} x+j\left({ }^{\vee} x+j\left(x+j\left(y^{\prime}\right)\right)\right)={ }^{\vee}{ }^{\vee} x+j\left({ }^{\vee} x+j\left(x+j\left(x^{\vee}+y\right)\right)\right),
$$

and since $\tilde{R}_{x}$ is a group homomorphism and by (1.4.1),

$$
\tilde{R}_{x} y^{\prime}=\tilde{R}_{x}\left(x^{\vee}+y\right)={ }^{\vee \vee} x+\tilde{R}_{x} y .
$$

Comparison yields, by (1.2.2),

$$
\tilde{R}_{x} y=j\left({ }^{\vee} x+j\left(x+j\left(x^{\vee}+y\right)\right)\right)=R_{x} y .
$$

1.5 Moufang sets and the functor $\mathbb{D}$. A Moufang set $[15,4.4]$ is a pair $M=$ $(X, \mathscr{U})$ where $X$ is a non-empty set and $\mathscr{U}$ is a map from $X$ into the set of all subgroups of the symmetric group $\operatorname{Sym}(X)$ of $X$ satisfying, for all $p \in X$,

(M1) $\mathscr{U}(p)$ acts simply transitively on $X-\{p\}$ (and therefore fixes $p$ ),

(M2) $\mathscr{U}(p)$ normalizes the set $\mathscr{U}(X)$.

The little projective group of $M$ is $\mathrm{G}(M)=\langle\mathscr{U}(p): p \in X\rangle \subset \operatorname{Sym}(X)$. Axiom (M2) is equivalent to the equivariance of $\mathscr{U}$ with respect to $G(M)[9,(2.4 .1)]$ :

$$
\mathscr{U}(g \cdot p)=g \circ \mathscr{U}(p) \circ g^{-1},
$$

for all $g \in \mathrm{G}(M)$ and $p \in X$.

A basis of $M$ is a pair $b=\left(b^{+}, b^{-}\right)$of distinct points of $X[9,2.6]$, and a based Moufang set is Moufang set with a choice of basis.

Let $(M, b)$ be a based Moufang set and put $U^{\sigma}=\mathscr{U}\left(b^{\sigma}\right)$. By [9, Lemma 3.1], for all $x \in \dot{U}^{\sigma}$ there exists a unique $y=j_{\sigma}(x) \in \dot{U}^{-\sigma}$ such that

$$
x \cdot b^{-\sigma}=j_{\sigma}(x) \cdot b^{\sigma} .
$$

We put $\mathbb{D}(M, b)=\left(\left(U^{+}, U^{-}\right),\left(j_{+}, j_{-}\right)\right)$. Then the main result of [9] is 
1.6 Theorem ([9, Theorem 3.5]). $\mathbb{D}$ is a functor from based Moufang sets to division pairs which is an equivalence of categories.

In particular, for every division pair $V$ there exists a based Moufang set $(M, b)$ with $\mathbb{D}(M, b) \cong V$, unique up to isomorphism.

A functor $\mathbb{P}$ from division pairs to based Moufang sets, essentially an inverse of $\mathbb{D}$, is constructed explicitly in [9, Section 3]. The details will not be needed here.

\section{The division pair of a rank one group}

2.1 Rank one groups. A rank one group is a triple $\mathscr{G}=\left(G, U^{+}, U^{-}\right)$consisting of a group $G$ and a pair of subgroups $\left(U^{+}, U^{-}\right)$, called root groups, such that

(R0) $G$ is generated by $U^{+}$and $U^{-}$,

(R1) $U^{+} \cap U^{-}=\{1\}$,

(R2) for all $\sigma \in\{+,-\}$ and all $x \in \dot{U}^{\sigma}:=U^{\sigma}-\{1\}$ there exists $y \in U^{-\sigma}$ with the property that

$$
\operatorname{Int}(x) U^{-\sigma}=\operatorname{Int}(y) U^{\sigma} .
$$

This definition is a generalization of Timmesfeld's [12, I, (1.1)] inasmuch as we do not assume the root groups to be nilpotent nor to be non-trivial. The nilpotence condition is not required for the elementary facts with which we are concerned here.

A morphism $\varphi:\left(G, U^{+}, U^{-}\right) \rightarrow\left(G^{\prime}, U^{\prime+}, U^{\prime-}\right)$ of rank one groups is a group homomorphism $\varphi: G \rightarrow G^{\prime}$ mapping $U^{\sigma}$ injectively to $U^{\prime \sigma}$, for $\sigma \in\{+,-\}$. Homomorphisms of this type are closed under composition, so rank one groups form a category, denoted 1-grp.

The requirement that $\varphi$ be injective on the root groups only excludes trivial homomorphisms. Indeed, assume $1 \neq x \in U^{\sigma} \cap \operatorname{Ker}(\varphi)$ for $\sigma=+$ or $\sigma=-$, and let $y \in U^{-\sigma}$ as in (2.1.1), so $U^{\sigma}=\operatorname{Int}\left(y^{-1} x\right) U^{-\sigma}$. Applying $\varphi$ yields $\varphi\left(U^{\sigma}\right)=$ $\operatorname{Int}\left(\varphi(y)^{-1}\right) \varphi\left(U^{-\sigma}\right)=\varphi\left(\operatorname{Int}\left(y^{-1}\right) U^{-\sigma}\right)=\varphi\left(U^{-\sigma}\right) \subset U^{\prime+} \cap U^{\prime-}=\{1\}$ by (R1), so $U^{\sigma} \subset \operatorname{Ker}(\varphi)$. Since $U^{-\sigma}$ is conjugate to $U^{\sigma}$ by $(\mathrm{R} 2)$, we have $U^{-\sigma} \subset \operatorname{Ker}(\varphi)$ as well, so $G=\operatorname{Ker}(\varphi)$ by (R0).

If $\mathscr{G}$ is a rank one group then so is $\mathscr{G}$ op $=\left(G, U^{-}, U^{-}\right)$, called the opposite of $\mathscr{G}$. Putting $\varphi^{\mathrm{op}}=\varphi$ for a morphism of rank one groups, we obtain a functor ()$^{\mathrm{op}}:$ 1-grp $\rightarrow$ 1-grp whose square is the identity.

In the remainder of this section, we collect a number of facts on rank one groups. Perhaps with the exception of the statements concerning morphisms, these are mostly well known, see [12, Chapter I], [1, 7.8.2]. For the convenience of the reader, and since our notation and terminology differs from these sources, proofs are included. The following lemma is essentially [12, I, Lemma (1.2), (2), (3)].

2.2 Lemma. For a rank one group $\mathscr{G}=\left(G, U^{+}, U^{-}\right)$, put $B^{\sigma}=\operatorname{Norm}_{G}\left(U^{\sigma}\right)$ and $H=B^{+} \cap B^{-}$.

(a) $U^{\sigma} \cap B^{-\sigma}=\{1\}$ and $\mathscr{Z}(G) \subset H$. 
(b) For $x \in \dot{U}^{\sigma}$, the element $y \in U^{-\sigma}$ satisfying (2.1.1) is uniquely determined and belongs to $\dot{U}^{-\sigma}$. We write $y=j_{\sigma}(x)$ and then have the defining relation

$$
\operatorname{Int}(x) U^{-\sigma}=\operatorname{Int}\left(j_{\sigma}(x)\right) U^{\sigma} .
$$

(c) The map $j_{\sigma}: \dot{U}^{\sigma} \rightarrow \dot{U}^{-\sigma}$ is bijective, with inverse map

$$
j_{\sigma}^{-1}=j_{-\sigma}
$$

Proof. (a) Assume $x \in \dot{U}^{\sigma}$ normalizes $U^{-\sigma}$ and choose $y \in U^{-\sigma}$ as in (2.1.1). Then $U^{-\sigma}=\operatorname{Int}(x) U^{-\sigma}=\operatorname{Int}(y) U^{\sigma}$ and hence $U^{\sigma}=\operatorname{Int}\left(y^{-1}\right) U^{-\sigma}=U^{-\sigma}$, so $1 \neq x \in U^{+} \cap U^{-}$, contradicting (R1). A central element normalizes $U^{+}$and $U^{-}$, hence belongs to $B^{+} \cap B^{-}=H$.

(b) Assume $\operatorname{Int}(x) U^{-\sigma}=\operatorname{Int}(y) U^{\sigma}=\operatorname{Int}(v) U^{\sigma}$ for $y, v \in U^{-\sigma}$. Then $v^{-1} y$ normalizes $U^{\sigma}$, so $v^{-1} y \in U^{-\sigma} \cap B^{\sigma}=\{1\}$ by (a). We also have $y \neq 1$ else $\operatorname{Int}(x) U^{-\sigma}=U^{\sigma}$ which implies $U^{-\sigma}=\operatorname{Int}\left(x^{-1}\right) U^{\sigma}=U^{\sigma}$ and $1 \neq x \in U^{+} \cap U^{-}$, contradicting (R1).

(c) Put $y=j_{\sigma}(x)$. By (2.2.1), with $\sigma$ replaced by $-\sigma$ and $x$ replaced by $y$, we have

$$
\operatorname{Int}(y) U^{\sigma}=\operatorname{Int}\left(j_{-\sigma}(y)\right) U^{-\sigma} \text {. }
$$

By comparing this with (2.2.1) and using (b), it follows that

$$
x=j_{-\sigma}(y)=j_{-\sigma}\left(j_{\sigma}(x)\right) .
$$

Since $\sigma$ can be + and - , this shows that $j_{\sigma}$ is bijective with inverse map $j_{-\sigma}$.

To simplify notation, we will often drop the index $\sigma$ at $j_{\sigma}$ when it can be restored from the context. We collect next a number of standard properties of rank one groups, see also $[12, \S 1, \S 2]$. Let

$$
\Xi=\left\{\operatorname{Int}(g) U^{+}: g \in G\right\} \subset \operatorname{sbgr}(G)
$$

be the set of all conjugates of $U^{+}$. By (R2), $U^{+}$and $U^{-}$are conjugate, thus $U^{-} \in \Xi$ and $\Xi$ is also the set of conjugates of $U^{-}$. We will often use the notation

$$
g \cdot U=\operatorname{Int}(g) U=g U g^{-1}
$$

for the action of $G$ on $\Xi$, and write $o^{\sigma}=U^{\sigma}$ when we consider $U^{\sigma}$ as a "point" of $\Xi$. Then $\Xi$ is the orbit of $o^{+}$or $o^{-}$under $G$ and $B^{\sigma}$ is the isotropy group of $o^{\sigma}$. In particular,

$$
x \cdot o^{\sigma}=o^{\sigma} \quad \text { for } x \in U^{\sigma},
$$

while formula (2.2.1) says

$$
x \cdot o^{-\sigma}=j(x) \cdot o^{\sigma} \quad \text { for } x \in \dot{U}^{\sigma} .
$$

We also define subsets $\Omega^{\sigma}$ of $G$ by

$$
\Omega^{\sigma}=U^{-\sigma} H U^{\sigma} \subset G .
$$

Part (a) and (b) of the following lemma is [12, I, Lemma (1.2)(1), Proposition (1.3)]. 
2.3 Lemma. Let $\left(G, U^{+}, U^{-}\right)$be a rank one group and assume $G \neq\{1\}$, equivalently, $U^{\sigma} \neq\{1\}$.

(a) For $\sigma \in\{+,-\}$,

$$
\Xi=\left\{o^{\sigma}\right\} \dot{\cup}\left\{x \cdot o^{-\sigma}: x \in U^{\sigma}\right\}
$$

(disjoint union). The group $U^{\sigma}$, acting on $\Xi$ as in (2.2.4), fixes $o^{\sigma}$ and is simply transitive on $\Xi-\left\{o^{\sigma}\right\}$.

(b) G acts doubly transitively on $\Xi$.

(c) The elements of $\Xi$ have the following intersection property: for $U_{1}, U_{2} \in \Xi$, either $U_{1}=U_{2}$ or $U_{1} \cap U_{2}=\{1\}$.

(d) $B^{\sigma}=U^{\sigma} H \cong U^{\sigma} \rtimes H$ (semidirect product).

(e) The multiplication map $U^{-\sigma} \times H \times U^{\sigma} \rightarrow \Omega^{\sigma}$ is bijective. An element $g \in G$ belongs to $\Omega^{\sigma}$ if and only if $g \cdot o^{\sigma} \neq o^{-\sigma}$, and

$$
G=U^{\sigma} \Omega^{\sigma}
$$

Proof. (a) If the union in (2.3.1) were not disjoint then $U^{\sigma}=o^{\sigma}=x \cdot o^{-\sigma}=$ $\operatorname{Int}(x) U^{-\sigma}$ for some $x \in U^{\sigma}$. This would imply $U^{-\sigma}=\operatorname{Int}\left(x^{-1}\right) U^{\sigma}=U^{\sigma}$, hence $U^{+}=U^{-}=\{1\}$ by (R1) and therefore $G=\{1\}$ by (R0), contradiction.

Let $\Xi^{\prime}$ denote the right hand side of (2.3.1). Then $\Xi^{\prime} \subset \Xi$, so to prove equality it suffices to show that $\Xi^{\prime}$ is stable under G. Clearly, $\Xi^{\prime}$ is stable under $U^{\sigma}$. Now by (2.2.6) and Lemma 2.2(c),

$$
\begin{aligned}
\Xi^{\prime} & =\left\{o^{\sigma}\right\} \cup\left\{o^{-\sigma}\right\} \cup\left\{x \cdot o^{-\sigma}: x \in \dot{U}^{\sigma}\right\}=\left\{o^{\sigma}\right\} \cup\left\{o^{-\sigma}\right\} \cup\left\{y \cdot o^{\sigma}: y \in \dot{U}^{-\sigma}\right\} \\
& =\left\{o^{-\sigma}\right\} \cup\left\{y \cdot o^{\sigma}: y \in U^{-\sigma}\right\} .
\end{aligned}
$$

This shows that $\Xi^{\prime}$ is stable under $U^{-\sigma}$ as well, hence stable under $G$.

By (2.3.1), $\Xi-\left\{o^{\sigma}\right\}=\left\{x \cdot o^{-\sigma}: x \in U^{\sigma}\right\}$ is the orbit of $o^{\sigma}$ under $U^{\sigma}$. To show that $U^{\sigma}$ acts freely, suppose $z \cdot\left(x \cdot o^{-\sigma}\right)=x \cdot o^{-\sigma}$ for some $z \in U^{\sigma}$. Then $\left(x^{-1} z x\right) \cdot o^{-\sigma}=o^{-\sigma}$, so $x^{-1} z x \in U^{\sigma} \cap B^{-\sigma}=\{1\}$ by Lemma 2.2(a), whence $z=1$.

(b) To prove double transitivity, it suffices that the isotropy group of some point, say $o^{+}$, act transitively on $\Xi-\left\{o^{+}\right\}$. This follows from (a).

(c) Assume $U_{1}, U_{2} \in \Xi$ and $U_{1} \neq U_{2}$. By (b), there exists $g \in G$ such that $U_{1}=\operatorname{Int}(g) U^{+}$and $U_{2}=\operatorname{Int}(g) U^{-}$. Hence $U_{1} \cap U_{2}=\operatorname{Int}(g)\left(U^{+} \cap U^{-}\right)=\{1\}$ by (R1).

(d) Clearly, $U^{\sigma} H \subset B^{\sigma}$ and $U^{\sigma}$ is normal in $B^{\sigma}$ by its very definition. We have $U^{\sigma} \cap H \subset U^{\sigma} \cap B^{-\sigma}=\{1\}$ by Lemma 2.2(a). Finally, let $b \in B^{\sigma}$. Then $b$ (acting on $\Xi$ ) fixes $o^{\sigma}$, hence stabilizes $\Xi-\left\{\sigma^{\sigma}\right\}$. By (a), there exists $u \in U^{\sigma}$ such that $\operatorname{Int}(b) U^{-\sigma}=\operatorname{Int}(u) U^{-\sigma}$. It follows that $u^{-1} b$ normalizes $U^{-\sigma}$ and $U^{\sigma}$, so $u^{-1} b=h \in H$ and $b=u h \in U^{\sigma} H$.

(e) Assume $v h u=v^{\prime} h^{\prime} u^{\prime}$ for $u, u^{\prime} \in U^{\sigma}, h, h^{\prime} \in H$ and $v, v^{\prime} \in U^{-\sigma}$. Then $u\left(u^{\prime}\right)^{-1}=h^{-1} v^{-1} v^{\prime} h^{\prime} \in U^{\sigma} \cap B^{-\sigma}=\{1\}$ by Lemma 2.2(a), whence $u=u^{\prime}$ and $v^{-1} v^{\prime}=h\left(h^{\prime}\right)^{-1} \in U^{-\sigma} \cap B^{\sigma}=\{1\}$. 
If $g=v h u \in \Omega^{\sigma}$ then $g \cdot o^{\sigma}=v \cdot o^{\sigma}$ (since $o^{\sigma}$ is fixed under $B^{\sigma}=H U^{\sigma}$ ), and $v \cdot o^{\sigma} \neq o^{-\sigma}$ because $\Xi=\left\{o^{-\sigma}\right\} \dot{\cup} U^{-\sigma} \cdot o^{\sigma}$ by (2.3.1). Conversely, let $g \in G$ and assume $g \cdot o^{\sigma} \neq o^{-\sigma}$, thus $g \cdot o^{\sigma} \in U^{-\sigma} \cdot o^{\sigma}$, say, $g \cdot o^{\sigma}=v \cdot o^{\sigma}$ for some $v \in U^{-\sigma}$. Then $v^{-1} g \cdot o^{\sigma}=o^{\sigma}$ whence $v^{-1} g \in B^{\sigma}$ and $g \in v B^{\sigma} \subset \Omega^{\sigma}$.

Finally, let $g \notin \Omega^{\sigma}$ so that $g \cdot o^{\sigma}=o^{-\sigma}$. Choose any $u \in \dot{U}^{\sigma}$. Then $u^{-1} g$. $o^{\sigma}=u^{-1} \cdot o^{-\sigma} \neq o^{-\sigma}$ since $U^{\sigma}$ acts freely on $\Xi-\left\{o^{\sigma}\right\}$. Hence $u^{-1} g \in \Omega^{\sigma}$, so $g \in u \Omega^{\sigma} \subset U^{\sigma} \Omega^{\sigma}$.

2.4 Proposition. Let $\varphi: \mathscr{G}=\left(G, U^{+}, U^{-}\right) \rightarrow \mathscr{G}^{\prime}=\left(G^{\prime}, U^{\prime+}, U^{\prime-}\right)$ be a morphism of rank one groups. We use the notation introduced in Lemma 2.2 for $\mathscr{G}^{\prime}$ as well, so $B^{\prime \sigma}=\operatorname{Norm}_{G^{\prime}}\left(U^{\prime \sigma}\right)$ etc.

(a) $\varphi$ is compatible with the maps $j_{\sigma}$ in the sense that the diagrams

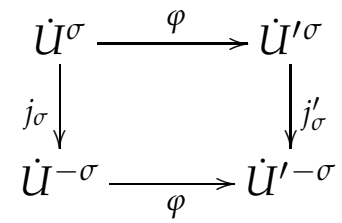

are commutative.

(b) $\varphi^{-1}\left(B^{\prime \sigma}\right)=B^{\sigma}, \varphi^{-1}\left(H^{\prime}\right)=H$ and $\varphi^{-1}\left(\Omega^{\prime \sigma}\right)=\Omega^{\sigma}$.

(c) $\varphi^{-1}\left(\mathscr{Z}\left(G^{\prime}\right)\right) \subset \mathscr{Z}(G)$; in particular, the kernel of $\varphi$ is central and contained in H. Moreover, $\varphi^{-1}\left(U^{\prime \sigma}\right)=\operatorname{Ker}(\varphi) U^{\sigma}$.

Proof. (a) Let $x \in \dot{U}^{\sigma}$ and $y=j_{\sigma}(x) \in U^{-\sigma}$. Put $Y^{\sigma}:=\varphi\left(U^{\sigma}\right)$, so that $\{1\} \neq$ $Y^{\sigma} \subset U^{\prime \sigma}$. By applying $\varphi$ to (2.1.1), we obtain

$$
\operatorname{Int}(\varphi(x)) Y^{-\sigma}=\operatorname{Int}(\varphi(y)) Y^{\sigma}
$$

Hence the subgroups $\operatorname{Int}(\varphi(x)) U^{\prime-\sigma}$ and $\operatorname{Int}(\varphi(y)) U^{\prime \sigma}$ have nontrivial intersection. By the intersection property (Lemma 2.3(c)), they are equal. Now $\varphi(y)=$ $\varphi\left(j_{\sigma}(x)\right)=j_{\sigma}^{\prime}(\varphi(x))$ follows from Lemma 2.2(b).

(b) If $G=\{1\}$ this is trivial so we may assume $G \neq\{1\}$, that is, $U^{\sigma} \neq$ $\{1\}$. We first show $B^{\sigma} \subset \varphi^{-1}\left(B^{\prime \sigma}\right)$. Let $b \in B^{\sigma}$. Then $\operatorname{Int}(b) U^{\sigma}=U^{\sigma}$ implies $\operatorname{Int}(\varphi(b)) Y^{\sigma}=Y^{\sigma}$. Hence, $U^{\prime \sigma}$ and $\operatorname{Int}(\varphi(b)) U^{\prime \sigma}$ have non-trivial intersection. By the intersection property these groups are equal, so $\varphi(b)$ normalizes $U^{\prime \sigma}$, that is, $\varphi(b) \in B^{\prime \sigma}$. This implies $\varphi(H)=\varphi\left(B^{+} \cap B^{-}\right) \subset \varphi\left(B^{+}\right) \cap \varphi\left(B^{-}\right) \subset B^{\prime+} \cap$ $B^{\prime-}=H^{\prime}$ and also $\varphi\left(\Omega^{\sigma}\right) \subset \Omega^{\prime \sigma}$.

For the reverse inclusions, we first show $\varphi^{-1}\left(\Omega^{\prime \sigma}\right) \subset \Omega^{\sigma}$. Let $g \in \varphi^{-1}\left(\Omega^{\prime \sigma}\right)$ and assume, aiming for a contradiction, that $g \notin \Omega^{\sigma}$. Then by Lemma 2.3(e), $g \cdot o^{\sigma}=o^{-\sigma}$, that is, $\operatorname{Int}(g) U^{\sigma}=U^{-\sigma}$. Applying $\varphi$ yields $\operatorname{Int}(\varphi(g)) Y^{\sigma}=Y^{-\sigma}$ and shows that the groups $\operatorname{Int}(\varphi(g)) U^{\prime \sigma}$ and $U^{\prime-\sigma}$ have non-trivial intersection and therefore are equal. This says $\varphi(g) \cdot o^{\prime \sigma}=o^{\prime-\sigma}$, so by Lemma 2.3(e), $\varphi(g) \notin \Omega^{\prime \sigma}$, contradiction.

Now let $g \in \varphi^{-1}\left(B^{\prime \sigma}\right)$. Then $g \in \varphi^{-1}\left(\Omega^{\prime \sigma}\right)=\Omega^{\sigma}$, so $g=v h u \in U^{-\sigma} H U^{\sigma}$, and it remains to show that $v=1$. But $\varphi(g)=\varphi(v) \varphi(h) \varphi(u) \in B^{\prime \sigma}=H^{\prime} U^{\prime \sigma}$, so 
by Lemma 2.3(e), $\varphi(v)=1$ which implies $v=1$ since $\varphi$ is injective on the root groups. Finally, $\varphi^{-1}\left(H^{\prime}\right)=\varphi^{-1}\left(B^{\prime+} \cap B^{\prime-}\right)=B^{+} \cap B^{-}=H$.

(c) Let $\pi(g) \in \mathscr{Z}\left(G^{\prime}\right)$. Since $\mathscr{Z}\left(G^{\prime}\right) \subset H^{\prime}$ by Lemma 2.2(a), we have $g \in \pi^{-1}\left(H^{\prime}\right)=H$ by (b). Now $H$ normalizes $U^{\sigma}$, so $\left(\left(g, U^{\sigma}\right)\right) \subset U^{\sigma}$. By applying $\varphi$, we obtain $\varphi\left(\left(g, U^{\sigma}\right)\right) \subset\left(\left(\varphi(g), U^{\prime \sigma}\right)\right)=\{1\}$ since $\varphi(g)$ is central in $G^{\prime}$. As $\varphi$ is injective on $U^{\sigma}$, it follows that $g$ commutes with $U^{\sigma}$ and therefore is central in $G$ by $(\mathrm{R} 0)$.

Finally, $g \in \varphi^{-1}\left(U^{\prime \sigma}\right) \subset \varphi^{-1}\left(B^{\prime \sigma}\right)=B^{\sigma}$ implies, by Lemma 2.3(d), that $g=h x \in H U^{\sigma}$ and $\varphi(g)=\varphi(h) \varphi(x) \in U^{\prime \sigma}$, hence $\varphi(h)=1$ so $g \in \operatorname{Ker}(\varphi) U^{\sigma}$. The opposite inclusion $\operatorname{Ker}(\varphi) U^{\sigma} \subset \varphi^{-1}\left(U^{\prime \sigma}\right)$ is clear.

2.5 The functor $\Delta$. For a rank one group $\mathscr{G}=\left(G, U^{+}, U^{-}\right)$we put

$$
\Delta(\mathscr{G})=\left(\left(U^{+}, U^{-}\right),\left(j_{+}, j_{-}\right)\right) .
$$

Then Lemma 2.2 says that $\Delta(\mathscr{G})$ is a pre-division pair in the sense of 1.1. If $\varphi: \mathscr{G} \rightarrow \mathscr{G}^{\prime}$ is a morphism of rank one groups, Proposition 2.4(a) shows that

$$
\Delta(\varphi)=\left(\varphi\left|U^{+}, \varphi\right| U^{-}\right): \Delta(\mathscr{G}) \rightarrow \Delta\left(\mathscr{G}^{\prime}\right)
$$

is a morphism of pre-division pairs. It is immediately verified that this defines a functor $\Delta:$ 1-grp $\rightarrow$ pre-div, and it is clear that $\Delta$ commutes with ( ) op. The conventions of 1.1 will be applied to $\Delta(\mathscr{G})$; in particular, the group law in $U^{\sigma}$ will often be written additively. We show next that $\Delta$ takes values in the category div of division pairs.

2.6 Lemma. For $x \in \dot{U}^{\sigma}$, let ${ }^{\vee} x=i(j(x))=-j(x)$ and $x^{\vee}=j(i(x))=j(-x)$ as in (1.1.3), and put

$$
w_{x}={ }^{\vee} x x x^{\vee} \in U^{-\sigma} U^{\sigma} U^{-\sigma},
$$

called the Weyl element determined by $x$. Then

$$
w_{-x}=w_{x}^{-1}
$$

and $w_{x}$ acting on $\Xi$ switches $o^{\sigma}$ and $o^{-\sigma}$ :

$$
w_{x} \cdot o^{-\sigma}=o^{\sigma}, \quad w_{x} \cdot o^{\sigma}=o^{-\sigma} ;
$$

equivalently,

$$
\operatorname{Int}\left(w_{x}\right) U^{-\sigma}=U^{\sigma}, \quad \operatorname{Int}\left(w_{x}\right) U^{\sigma}=U^{-\sigma}
$$

For a morphism $\varphi: \mathscr{G} \rightarrow \mathscr{G}^{\prime}$ of rank one groups, we have

$$
\varphi\left(w_{x}\right)=w_{\varphi(x)} .
$$

Proof. From (1.1.5) it follows easily that $w_{-x}=w_{x}^{-1}$. By (2.2.6) and (2.2.5), we have

$$
\begin{aligned}
w_{x} \cdot o^{-\sigma} & =\left({ }^{\vee} x\right) \cdot\left(x \cdot o^{-\sigma}\right)={ }^{\vee} x \cdot\left(j(x) \cdot o^{\sigma}\right) \\
& =\left({ }^{\vee} x+j(x)\right) \cdot o^{\sigma}=(-j(x)+j(x)) \cdot o^{\sigma}=o^{\sigma} .
\end{aligned}
$$

This implies $o^{-\sigma}=w_{x}^{-1} \cdot o^{\sigma}=w_{-x} \cdot o^{\sigma}$, so replacing $x$ by $-x$ shows $w_{x} \cdot o^{\sigma}=o^{-\sigma}$ as well. Since $\Delta(\varphi)$ is a morphism of pre-division pairs, (1.1.6) shows $\varphi\left(x^{\vee}\right)=$ $\varphi(x)^{\vee}$ and $\varphi\left({ }^{\vee} x\right)={ }^{\vee} \varphi(x)$. This implies (2.6.4). 
2.7 Proposition. Let $\mathscr{G}=\left(G, U^{+}, U^{-}\right)$be a rank one group and let $\Delta(\mathscr{G})=$ $\left(U^{+}, U^{-}\right)$be the associated pre-division pair. For $x \in \dot{U}^{\sigma}$ let $R_{x}: U^{-\sigma} \rightarrow U^{\sigma}$ be as in Lemma 1.2(a). Then $R_{x}$ is given by conjugation with $w_{x}$ :

$$
w_{x} y w_{x}^{-1}=R_{x} y
$$

Hence $R_{x}$ is a group homomorphism. Since this is true for all $x \in \dot{U}^{\sigma}$, it follows that $\Delta(\mathscr{G})$ is a division pair, so $\Delta$ is a functor from rank one groups to division pairs.

Remark. For Moufang sets, formula (2.7.1) is just a reformulation of the wellknown fact that $\left(\alpha_{y}^{\tau}\right)^{\mu_{x}}=\alpha_{y h_{x}}$.

Proof. Let $y \in U^{-\sigma}$ and $x \in \dot{U}^{\sigma}$. By (2.6.3), $w_{x} y w_{x}^{-1} \in U^{\sigma}$. If $y=0$ then $R_{x} y=0=$ $w_{x} y w_{x}^{-1}$, so we may assume $y \neq 0$. By Lemma 2.3(a), $U^{\sigma}$ acts freely on $\boldsymbol{E}-\left\{o^{\sigma}\right\}$ and we have $o^{-\sigma} \in \Xi-\left\{o^{\sigma}\right\}$. Hence, if $u, u^{\prime} \in U^{\sigma}$ with $u \cdot o^{-\sigma}=u^{\prime} \cdot o^{-\sigma}$, then $u=u^{\prime}$. We apply this to $u=w_{x} y w_{x}^{-1}$ and $u^{\prime}=R_{x} y$ and distinguish two cases.

Case 1: $x^{\vee}+y=0$. Then, using repeatedly (2.2.6) and (2.6.2),

$$
\begin{aligned}
w_{x} y w_{x}^{-1} \cdot o^{-\sigma} & =w_{x} y \cdot\left(w_{x}^{-1} \cdot o^{-\sigma}\right)=w_{x} \cdot\left(y \cdot o^{\sigma}\right) \\
& =\left({ }^{\vee} x x\left(x^{\vee}+y\right)\right) \cdot o^{\sigma}={ }^{\vee} x \cdot\left(x \cdot o^{\sigma}\right) \\
& ={ }^{\vee} x \cdot o^{\sigma}=j\left({ }^{\vee} x\right) \cdot o^{-\sigma}=\left(R_{x} y\right) \cdot o^{-\sigma} .
\end{aligned}
$$

Case 2: $x^{\vee}+y \neq 0$. First note that this implies $x+j\left(x^{\vee}+y\right) \neq 0$ as well. Indeed, otherwise $-x=j\left(x^{\vee}+y\right)$, whence $x^{\vee}+y=j(-x)=x^{\vee}$ and therefore $y=0$, which is impossible. Now compute:

$$
\begin{aligned}
w_{x} y w_{x}^{-1} \cdot o^{-\sigma} & =w_{x} y \cdot\left(w_{x}^{-1} \cdot o^{-\sigma}\right)=w_{x} y \cdot o^{\sigma} \\
& \left.=\left({ }^{\vee} x x\right) \cdot\left(\left(x^{\vee}+y\right) \cdot o^{\sigma}\right)=\left({ }^{\vee} x x\right) \cdot\left(j\left(x^{\vee}+y\right)\right) \cdot o^{-\sigma}\right) \\
& ={ }^{\vee} x \cdot\left(\left(x+j\left(x^{\vee}+y\right)\right) \cdot o^{-\sigma}\right)={ }^{\vee} x \cdot\left(j\left(x+j\left(x^{\vee}+y\right)\right) \cdot o^{\sigma}\right) \\
& =\left({ }^{\vee} x+j\left(x+j\left(x^{\vee}+y\right)\right)\right) \cdot o^{\sigma}=j\left({ }^{\vee} x+j\left(x+j\left(x^{\vee}+y\right)\right) \cdot o^{-\sigma}\right. \\
& =\left(R_{x} y\right) \cdot o^{-\sigma} .
\end{aligned}
$$

2.8 Coverings. A covering of a rank one group $\mathscr{G}=\left(G, U^{+}, U^{-}\right)$is a morphism $\varphi: \tilde{\mathscr{G}}=\left(\tilde{G}, \tilde{U}^{+}, \tilde{U}^{-}\right) \rightarrow \mathscr{G}$ of rank one groups satisfying the following equivalent conditions:

(i) $\varphi: \tilde{U}^{\sigma} \rightarrow U^{\sigma}$ is bijective for $\sigma= \pm$,

(ii) $\Delta(\varphi): \Delta(\tilde{\mathscr{G}}) \rightarrow \Delta(\mathscr{G})$ is an isomorphism,

(iii) $\varphi: \tilde{G} \rightarrow G$ is surjective.

In the terminology of $[13,2.5], \tilde{\mathscr{G}}$ is a rank one extension of $\mathscr{G}$.

We have (i) $\Longleftrightarrow$ (ii) because $\Delta(\varphi)=\left(\varphi\left|U^{+}, \varphi\right| U^{-}\right)$, and (i) $\Longrightarrow$ (iii) is clear from (R0). For (iii) $\Longrightarrow$ (i), let $\varphi: \tilde{G} \rightarrow G$ be surjective and let $x \in U^{\sigma}$. Then $x=\varphi(g)$ for some $g \in \varphi^{-1}\left(U^{\sigma}\right)=\operatorname{Ker}(\varphi) \tilde{U}^{\sigma}$ by Proposition 2.4(c), say, $g=k \tilde{x}$. 
Hence $x=\varphi(g)=\varphi(\tilde{x}) \in \varphi\left(\tilde{U}^{\sigma}\right)$, so $\varphi: \tilde{U}^{\sigma} \rightarrow U^{\sigma}$ is surjective, and it is injective by definition of morphism.

Let $\varphi: \tilde{\mathscr{G}} \rightarrow \mathscr{G}$ be a covering. Then the centres of $\tilde{G}$ and $G$ are related by

$$
\varphi^{-1}(\mathscr{Z}(G))=\mathscr{Z}(\tilde{G}), \quad \varphi(\mathscr{Z}(\tilde{G}))=\mathscr{Z}(G) .
$$

Indeed, since $\varphi$ is surjective, we have $\varphi(\mathscr{Z}(\tilde{G})) \subset \mathscr{Z}(G)$ and therefore $\mathscr{Z}(\tilde{G}) \subset$ $\varphi^{-1}(\mathscr{Z}(G))$. Since $\varphi^{-1}(\mathscr{Z}(G)) \subset \mathscr{Z}(\tilde{G})$ by Proposition $2.4(\mathrm{c})$, this proves the first equation. For the second, it remains to show $\mathscr{Z}(G) \subset \varphi(\mathscr{Z}(\tilde{G}))$. This follows from $\varphi^{-1}(\mathscr{Z}(G)) \subset \mathscr{Z}(\tilde{G})$ and surjectivity of $\varphi$.

If $\varphi: \tilde{\mathscr{G}} \rightarrow \mathscr{G}$ is a covering then by Proposition 2.4(c), G is (isomorphic to) a central quotient of $\tilde{G}$. The converse holds as well:

2.9 Lemma. Let $\mathscr{G}=\left(G, U^{+}, U^{-}\right)$be a rank one group. Let $K$ be a central subgroup of $G$, let $\pi: G \rightarrow \bar{G}=G / K$ be the canonical map and put $\bar{U}^{\sigma}=\pi\left(U^{\sigma}\right)$. Then $\overline{\mathscr{G}}=\left(\bar{G}, \bar{U}^{+}, \bar{U}^{-}\right)$is a rank one group, and $\pi: \mathscr{G} \rightarrow \overline{\mathscr{G}}$ is a covering. The centre of $\bar{G}$ is $\mathscr{Z}(G) / K$; in particular, $G / \mathscr{Z}(G)$ has trivial centre.

Proof. We verify the axioms of a rank one group for $\overline{\mathscr{G}}$. Clearly, $G=\left\langle U^{+}, U^{-}\right\rangle$ implies $\bar{G}=\left\langle\bar{U}^{+}, \bar{U}^{-}\right\rangle$, so we have (R0). By Lemma 2.2(a), $K$, being central, is contained in $H$ and has trivial intersection with $U^{\sigma}$. Assume $\pi(x)=\pi(y) \in$ $\bar{U}^{+} \cap \bar{U}^{-}$, where $x \in U^{+}$and $y \in U^{-}$. Then $y=k x$ for some $k \in K$, so $y \in U^{-} \cap K U^{+} \subset U^{-} \cap B^{+}=\{1\}$ by Lemma 2.2(a). This proves (R1). Axiom (R2) for $\bar{G}$ follows immediately from (R2) for $G$ by applying $\pi: G \rightarrow \bar{G}$. Since $\pi$ is surjective, it is a covering by 2.8. The remaining statements follow from (2.8.1).

\section{The Steinberg group of a division pair}

3.1 Definition. Let $V$ be a division pair. The Steinberg group of $V$, denoted $\operatorname{St}(V)$, is the group presented by generators and relations as follows. The generators are all symbols $\hat{\mathrm{e}}_{\sigma}(z), z \in V^{\sigma}, \sigma \in\{+,-\}$. For $x \in \dot{V}^{\sigma}$, put

$$
\hat{\mathrm{w}}_{\sigma}(x)=\hat{\mathrm{e}}_{-\sigma}\left({ }^{\vee} x\right) \hat{\mathrm{e}}_{\sigma}(x) \hat{\mathrm{e}}_{-\sigma}\left(x^{\vee}\right)
$$

where ${ }^{\vee} x$ and $x^{\vee}$ are as in (1.1.3). Then the relations are

$$
\begin{aligned}
\hat{\mathrm{e}}_{\sigma}(u+z) & =\hat{\mathrm{e}}_{\sigma}(u) \hat{\mathrm{e}}_{\sigma}(z), \\
\hat{\mathrm{w}}_{\sigma}(x) \hat{\mathrm{e}}_{-\sigma}(y) \hat{\mathrm{w}}_{\sigma}(x)^{-1} & =\hat{\mathrm{e}}_{\sigma}\left(R_{x}(y)\right),
\end{aligned}
$$

for all $\sigma \in\{+,-\}, u, z \in V^{\sigma}, x \in \dot{V}^{\sigma}$ and $y \in V^{-\sigma}$. Here $R_{x}$ is as in Lemma 1.2(a).

Let $h: V \rightarrow V^{\prime}$ be a homomorphism of division pairs. Then it follows easily from (1.1.6) and (1.2.3) that $h$ induces a group homomorphism $\operatorname{St}(h): \operatorname{St}(V) \rightarrow$ $\operatorname{St}\left(V^{\prime}\right)$ uniquely determined by

$$
\operatorname{St}(h)\left(\hat{\mathrm{e}}_{\sigma}(x)\right)=\hat{\mathrm{e}}_{\sigma}^{\prime}\left(h_{\sigma}(x)\right)
$$

and hence satisfying

$$
\operatorname{St}(h)\left(\hat{\mathbf{w}}_{\sigma}(x)\right)=\hat{\mathbf{w}}_{\sigma}^{\prime}\left(h_{\sigma}(x)\right)
$$


for all $x \in V^{\sigma}, \sigma \in\{+,-\}$. Thus $\mathrm{St}$ is a functor from division pairs to groups.

To simplify notation, we introduce the abbreviations

$$
\hat{U}^{\sigma}=\hat{\mathrm{e}}_{\sigma}\left(V^{\sigma}\right), \quad \hat{\mathrm{G}}=\mathrm{St}(V) .
$$

3.2 Special cases. (a) Let $V$ be a Jordan division pair. By [9, Proposition 1.11], $V$ is a division pair with $j$-maps given by $j(x)=-x^{-1}$ where $x^{-1}$ is the inverse (in the Jordan sense) of $x \in \dot{V}^{\sigma}$. Then the Steinberg group defined here is precisely the group introduced in $[8,1.4]$. This follows easily from $[8$, Corollary 1.8]. We will show in 3.9 that the group $\bar{G}$ of 3.4 and the projective elementary group of $V$ in the sense of $[10,1.1]$ are isomorphic.

(b) In [14], Timmesfeld defines Steinberg groups in the following setting. A $K$-structure consists of an abelian group $K$ and a map $f$ from $\dot{K}$ to the automorphism group of $K$, written $t \mapsto f_{t}$, satisfying the following axioms:

1. the map $\chi: t \mapsto f_{t}(t)$ is a bijection of $\dot{K}$ onto itself,

2. $\chi^{2}=\mathrm{Id}$,

3. $\chi(-x)=-\chi(x)$ for all $x \in \dot{K}$,

4. for all $x \neq y$ in $\dot{K}$,

$$
f_{x}(y)=\left[x+\left(y^{-1}-x^{-1}\right)^{-1}\right]^{-1}-x^{-1}
$$

where $z^{-1}:=-\chi(z)$

We now show that $K$-structures are in bijection with a particular class of division pairs. Hence, the group $\mathrm{St}_{2}\left(K, f_{t}\right)$ of [14, Theorem (2.1), Definition (2.2)] appears as a special case of our $\operatorname{St}(V)$.

Given a $K$-structure, define $V^{+}=V^{-}=K$ and $j_{+}=j_{-}=\chi$. By (1) and (2), the pair $V=\left(V^{+}, V^{-}\right)$with the maps $j_{\sigma}$ is a pre-division pair. We claim that the $R$-operators of $V$ are given by $R_{x}=-f_{x^{-1}}$, hence are group homomorphisms, and therefore $V$ is a division pair. By Lemma 1.4, it suffices to show this for the $\tilde{R}_{x}$. By (3), the $j_{\sigma}$ commute with group inversion, so we have ${ }^{\vee} x=x^{\vee}=x^{-1}$ and thus ${ }^{\vee \vee} x={ }^{\vee}\left(x^{\vee}\right)=x$ by (1.1.4). Let $0 \neq y \neq x^{-1}$. Then (1.4.2) reads

$$
\begin{aligned}
\tilde{R}_{x} y & =x+j\left({ }^{\vee} x+j(x+j(y))\right)=x-\left[x^{-1}-\left(x-y^{-1}\right)^{-1}\right]^{-1} \\
& =x-\left[x^{-1}+\left(y^{-1}-x\right)^{-1}\right]^{-1}=-f_{x^{-1}}(y) .
\end{aligned}
$$

For $y=x^{-1}$ we have, by (1.4.1), $\tilde{R}_{x} x^{-1}=x^{-1}$ while $f_{x^{-1}}\left(x^{-1}\right)=\chi\left(x^{-1}\right)=$ $\chi(-\chi(x))=-\chi^{2}(x)=-x$. Thus $R_{x}=-f_{x^{-1}}$, as asserted.

Conversely, let $V$ be a division pair with $V^{+}=V^{-}$abelian and $j_{+}=j_{-}=$: $\chi$, and assume that $\chi$ commutes with inversion. Define $x^{-1}=-\chi(x)$ and put $f_{x}(y)=-R_{x^{-1}} y$ for $x \in \dot{K}, y \in K$. Then it is easily seen that Timmesfeld's axioms are satisfied. Thus $K$-structures are the same as special abelian division pairs (cf. 4.13 and 4.14) which in addition satisfy $V^{+}=V^{-}$and $j_{+}=j_{-}$. 
The following lemma is essentially [12, I, Proposition (1.3)]. For the convenience of the reader we include a proof. See 1.5 for notation regarding Moufang sets.

3.3 Lemma. Let $M=(X, \mathscr{U})$ be a Moufang set with basis $b$, let $\bar{U}^{\sigma}=\mathscr{U}\left(b^{\sigma}\right)$ and let $\bar{G}=G(M)$ be the little projective group of $M$. Then $\overline{\mathscr{G}}(M, b)=\left(\bar{G}, \bar{U}^{+}, \bar{U}^{-}\right)$is a rank one group with trivial centre, and the division pairs associated with $(M, b)$ and $\overline{\mathscr{G}}(M, b)$ are the same:

$$
\Delta(\overline{\mathscr{G}}(M, b))=\mathbb{D}(M, b) .
$$

Proof. If $p \in X-\left\{b^{+}, b^{-}\right\}$then by (M1), there exists $u \in \bar{U}^{+}$such that $p=u \cdot b^{-}$, and therefore $\mathscr{U}(p)=u \circ \bar{U}^{-} \circ u^{-1} \in\left\langle\bar{U}^{+}, \bar{U}^{-}\right\rangle$by (1.5.1). This proves (R0). To show (R1), assume $u \in \bar{U}^{+} \cap \bar{U}^{-}$. Then $u \cdot b^{-}=b^{-}$since $\bar{U}^{-}$fixes $b^{-}$. But $\bar{U}^{+}$ acts freely on $X-\left\{b^{-}\right\}$and $u \in \bar{U}^{+}$, whence $u=1$. For (R2), let $x \in \bar{U}^{\sigma}-\{0\}$ and $y=j(x) \in \bar{U}^{-\sigma}$ as in (1.5.2). Then (1.5.1) shows

$$
x \circ \bar{U}^{-\sigma} \circ x^{-1}=\mathscr{U}\left(x \cdot b^{-\sigma}\right)=\mathscr{U}\left(j(x) \cdot b^{\sigma}\right)=j(x) \circ \bar{U}^{\sigma} \circ j(x)^{-1} .
$$

This proves (R2) and also shows that the $j$-maps for $\Delta(\overline{\mathscr{G}}(M, b))$, defined by (2.2.1), agree with the $j$-maps of $\mathbb{D}(M, b)$. So we have (3.3.1), and it remains to show that $\bar{G}$ has trivial centre. We may assume $\bar{G} \neq\{1\}$. Then the groups $\mathscr{U}(p)$ are non-trivial, and the map $\mathscr{U}: X \rightarrow \operatorname{sbgr}(\operatorname{Sym}(X))$ is injective. Indeed, assume $p \neq q \in X$ but $\mathscr{U}(p)=\mathscr{U}(q)$. We may take the pair $(p, q)=\left(b^{+}, b^{-}\right)$ as a basis of $M$ and then have $\bar{U}^{+}=\bar{U}^{-}$. Since $\bar{U}^{+} \cap \bar{U}^{-}=\{1\}$, it follows that $\bar{U}^{+}=\bar{U}^{-}=\{1\}$, contradicting $\bar{G} \neq\{1\}$. Now let $z \in \mathscr{Z}(\bar{G})$. Then $\mathscr{U}(z \cdot p)=z \circ \mathscr{U}(p) \circ z^{-1}=\mathscr{U}(p)$ (since $z$ is central in $\bar{G}$ ), hence $z \cdot p=p$ for all $p \in X$, showing $z=\operatorname{Id}_{X}=1_{\bar{G}}$.

3.4 Notation. By 1.6, the functor $\mathbb{D}$ from based Moufang sets to division pairs is an equivalence of categories. In particular, for every division pair $V$, there exists a based Moufang set $(M, b)$ such that $\mathbb{D}(M, b) \cong V$. In the sequel, we fix, for given $V \in$ div, such a Moufang set and an isomorphism

$$
\overline{\mathrm{e}}=\left(\overline{\mathrm{e}}_{+}, \overline{\mathrm{e}}_{-}\right): V \stackrel{\cong}{\longrightarrow} \mathbb{D}(M, b)
$$

of division pairs; for example, $(M, b)=\mathbb{P}(V)$ and $\overline{\mathrm{e}}=\eta_{V}$ as in [9, Proposition 3.4, Theorem 3.5]. Let $\bar{G}$ be the little projective group of $M$ and put $\bar{U}^{\sigma}=\mathscr{U}\left(b^{\sigma}\right)$ as in Lemma 3.3. By abuse of notation, we will write $\overline{\mathscr{G}}(V)=\overline{\mathscr{G}}(M, b)$. Then the maps

$$
\overline{\mathrm{e}}_{\sigma}: V^{\sigma} \stackrel{\cong}{\longrightarrow} \bar{U}^{\sigma}
$$

are group isomorphisms satisfying

$$
\overline{\mathrm{e}}_{-\sigma}(j(x))=j\left(\overline{\mathrm{e}}_{\sigma}(x)\right)
$$

and therefore, by the definition of the $j$-maps in $\Delta(\overline{\mathscr{G}}(V))$,

$$
\operatorname{Int}\left(\overline{\mathrm{e}}_{\sigma}(x)\right) \bar{U}^{-\sigma}=\operatorname{Int}\left(\overline{\mathrm{e}}_{-\sigma}(j(x))\right) \bar{U}^{\sigma}
$$


for all $x \in \dot{V}^{\sigma}, \sigma \in\{+,-\}$. We put

$$
\overline{\mathrm{w}}_{\sigma}(x)=\overline{\mathrm{e}}_{-\sigma}\left({ }^{\vee} x\right) \circ \overline{\mathrm{e}}_{\sigma}(x) \circ \overline{\mathrm{e}}_{-\sigma}\left(x^{\vee}\right) \in \bar{G},
$$

called the Weyl element determined by $x$. Since $\overline{\mathrm{e}}$ is a homomorphism of division pairs, it commutes with the maps $x \mapsto{ }^{\vee} x$ and $x \mapsto x^{\vee}$. Hence,

$$
\overline{\mathrm{w}}_{\sigma}(x)=w_{\overline{\mathrm{e}}_{\sigma}(x)}
$$

in the notation of 2.6.

3.5 Remark. The notation $\overline{\mathscr{G}}(V)$ might suggest that the assignment $V \mapsto \overline{\mathscr{G}}(V)$ defines a functor from division pairs to rank one groups. This is not the case because the little projective group of a Moufang set $M$ does not depend functorially on $M$. For example, let $M$ be the Moufang set of all isotropic lines with respect to the hermitian form $h$ with matrix $\left(\begin{array}{ll}0 & 1 \\ 1 & 0\end{array}\right)$ on $\mathbb{C}^{2}$ and let $M^{\prime}$ be the Moufang set of isotropic lines with respect to the hermitian form $h^{\prime}$ with matrix $\left(\begin{array}{lll}0 & 0 & 1 \\ 0 & 1 & 0 \\ 1 & 0 & 0\end{array}\right)$ on $\mathbb{C}^{3}$ (column vectors). Then there is a morphism $f: M \rightarrow M^{\prime}$ induced from $\left(\begin{array}{l}a \\ c\end{array}\right) \mapsto\left(\begin{array}{l}a \\ 0 \\ c\end{array}\right)$, but there is no homomorphism $\mathrm{G}(M) \rightarrow \mathrm{G}\left(M^{\prime}\right)$ compatible with $f$.

Indeed, the little projective group of $M$ is the projective unitary group $\mathrm{PU}(1,1)$ of the unitary group of $h$ while that of $M^{\prime}$ is $\mathrm{PU}(2,1)$, the projective unitary group of $h^{\prime}$. Denote by $[z]$ the line determined by a non-zero vector $z \in \mathbb{C}^{n}$. Then $\left[\begin{array}{l}a \\ c\end{array}\right] \in$ $M$ if and only if $\bar{a} c+\bar{c} a=0$, and $\left[\begin{array}{l}a \\ b \\ c\end{array}\right] \in M^{\prime}$ if and only if $\bar{a} c+\bar{c} a+|b|^{2}=0$. The morphism $f: M \rightarrow M^{\prime}$ is given by $\left[\begin{array}{l}a \\ c\end{array}\right] \mapsto\left[\begin{array}{l}a \\ 0 \\ c\end{array}\right]$. Consider the basis $o^{+}=\left[\begin{array}{l}1 \\ 0\end{array}\right]$ and $o^{-}=\left[\begin{array}{l}0 \\ 1\end{array}\right]$ of $M$ and put $b^{\prime \sigma}=f\left(o^{\sigma}\right)$. Then $f$ is a morphism of based Moufang sets, hence induces a group homomorphism $\partial_{o^{+}}(f): U^{+}=\mathscr{U}\left(o^{+}\right) \rightarrow U^{\prime+}=\mathscr{U}^{\prime}\left(o^{+}\right)$ by [9, Lemma 2.2]. In our case,

$$
U^{+}=\left[\begin{array}{cc}
1 & i \mathbb{R} \\
0 & 1
\end{array}\right] \subset \mathrm{PU}(1,1)
$$

$U^{\prime+}$ consist of all matrices

$$
\left[\begin{array}{lll}
1 & a & c \\
0 & 1 & b \\
0 & 0 & 1
\end{array}\right]
$$

where $a, b, c \in \mathbb{C}, \bar{a}+b=0$ and $a b=c+\bar{c}$, and $\partial_{o^{+}}(f)$ is given by

$$
\left[\begin{array}{cc}
1 & i r \\
0 & 1
\end{array}\right] \mapsto\left[\begin{array}{ccc}
1 & 0 & i r \\
0 & 1 & 0 \\
0 & 0 & 1
\end{array}\right]
$$

Here the square brackets indicate the projective transformations induced by the respective matrices, $r \in \mathbb{R}$ and $i=\sqrt{-1}$. Analogously, one has the homomorphism

$$
\partial_{o^{-}}(f):\left[\begin{array}{cc}
1 & 0 \\
i r & 1
\end{array}\right] \mapsto\left[\begin{array}{ccc}
1 & 0 & 0 \\
0 & 1 & 0 \\
i r & 0 & 1
\end{array}\right]
$$


Now consider the element $x=\left[\begin{array}{ll}1 & i \\ 0 & 1\end{array}\right] \in U^{+}$and the associated Weyl element

$$
w_{x}=\left({ }^{\vee} x\right) x\left(x^{\vee}\right)=\left[\begin{array}{ll}
1 & 0 \\
i & 1
\end{array}\right]\left[\begin{array}{ll}
1 & i \\
0 & 1
\end{array}\right]\left[\begin{array}{ll}
1 & 0 \\
i & 1
\end{array}\right]=\left[\begin{array}{ll}
0 & i \\
i & 0
\end{array}\right] \in U^{-} U^{+} U^{+} \subset \mathrm{G}(M) .
$$

Then $w_{x}^{2}=\left[\begin{array}{cc}-1 & 0 \\ 0 & -1\end{array}\right]=\operatorname{Id}_{M}$. Assume that the homomorphisms $\partial_{\sigma^{\sigma}}(f)$ extend to a homomorphism $\varphi: \mathrm{G}(M) \rightarrow \mathrm{G}\left(M^{\prime}\right)$. Then $\varphi\left(w_{x}\right)=w_{\varphi(x)}$ and hence $w_{\varphi(x)}^{2}=\mathrm{Id}$. But by a computation similar to the one above,

$$
w_{\varphi(x)}=\left[\begin{array}{lll}
0 & 0 & i \\
0 & 1 & 0 \\
i & 0 & 0
\end{array}\right]
$$

with square

$$
w_{\varphi(x)}^{2}=\left[\begin{array}{ccc}
-1 & 0 & 0 \\
0 & 1 & 0 \\
0 & 0 & -1
\end{array}\right] \neq \mathrm{Id}
$$

because it maps any $p=\left[\begin{array}{l}a \\ b \\ c\end{array}\right]$ with $b \neq 0$ to $\left[\begin{array}{c}-a \\ b \\ -c\end{array}\right] \neq p$, contradiction.

3.6 Lemma. Let $V$ be a division pair. We use the notation introduced in 3.1 and 3.4. There is a unique group homomorphism $\hat{\pi}: \hat{G} \rightarrow \bar{G}$ satisfying

$$
\hat{\pi}\left(\hat{\mathrm{e}}_{\sigma}(x)\right)=\overline{\mathrm{e}}_{\sigma}(x),
$$

for all $x \in V^{\sigma}$ and $\sigma \in\{+,-\}$. The homomorphism $\hat{\pi}$ is surjective and its restriction to $\hat{U}^{\sigma}$ is an isomorphism $\hat{U}^{\sigma} \stackrel{\cong}{\longrightarrow} \bar{U}^{\sigma}$. Hence $\hat{\mathrm{e}}_{\sigma}: V^{\sigma} \rightarrow \hat{U}^{\sigma}$ is an isomorphism as well.

Proof. We must show that the defining relations of $\operatorname{St}(V)$ hold for the $\overline{\mathrm{e}}_{\sigma}(x)$. This is clear for (3.1.2). Let us verify (3.1.3) in $\bar{G}$. As $\overline{\mathscr{G}}(V)$ is a rank one group, it follows from (3.4.6), (2.7.1) and the fact that $\overline{\mathrm{e}}$ is a homomorphism of division pairs that

$$
\operatorname{Int}\left(\overline{\mathbf{w}}_{\sigma}(x)\right) \overline{\mathrm{e}}_{-\sigma}(y)=R_{\overline{\mathrm{e}}_{\sigma}(x)} \overline{\mathrm{e}}_{-\sigma}(y)=\overline{\mathrm{e}}_{\sigma}\left(R_{x} y\right) .
$$

Hence the relations (3.1.3) hold in $\bar{G}$, with $\hat{\mathrm{e}}_{\sigma}$ and $\hat{\mathrm{w}}_{\sigma}$ replaced by $\overline{\mathrm{e}}_{\sigma}$ and $\overline{\mathrm{w}}_{\sigma}$, respectively. This proves the existence of $\hat{\pi}$. It is clear that $\hat{\pi}$ is uniquely determined by (3.6.1). The maps $\overline{\mathrm{e}}_{\sigma}: V^{\sigma} \rightarrow \bar{U}^{\sigma}$ being isomorphisms by (3.4.2), so are the $\hat{\mathrm{e}}_{\sigma}: V^{\sigma} \rightarrow \hat{U}^{\sigma}$. Since $\hat{G}$ and $\bar{G}$ are generated by the $\hat{U}^{\sigma}$ and $\bar{U}^{\sigma}$, respectively, $\hat{\pi}$ is surjective.

3.7 Theorem. (a) For a division pair $V$,

$$
\Sigma(V):=\left(\hat{\mathrm{G}}, \hat{U}^{+}, \hat{U}^{-}\right)=\left(\operatorname{St}(V), \hat{\mathrm{e}}_{+}\left(V^{+}\right), \hat{\mathrm{e}}_{-}\left(V^{-}\right)\right)
$$

is a rank one group, and for a morphism $h: V \rightarrow W$ of division pairs, $\Sigma(h):=\operatorname{St}(h)$ : $\Sigma(V) \rightarrow \Sigma(W)$ is a morphism of rank one groups. These assignments define a functor

$$
\Sigma: \operatorname{div} \rightarrow \text { 1-grp }
$$

from division pairs to rank one groups which commutes with ( ) ${ }^{\mathrm{op}}$. 
(b) The functor $\Sigma$ is left adjoint to the functor $\Delta: \mathbf{1}$-grp $\rightarrow \operatorname{div}$ of 2.5 and Proposition 2.7: for all $V \in \operatorname{div}$ and $\mathscr{G} \in \mathbf{1}$-grp, there are natural inverse bijections

$$
\operatorname{Mor}_{1-\operatorname{grp}}(\Sigma(V), \mathscr{G}) \underset{\beta}{\stackrel{\alpha}{\rightleftarrows}} \operatorname{Mor}_{\mathbf{d i v}}(V, \Delta(\mathscr{G})) \text {. }
$$

(c) For all $V \in \mathbf{d i v}$, the unit of the adjunction,

$$
\eta_{V}=\alpha\left(\operatorname{Id}_{\Sigma(V)}\right): V \rightarrow \Delta(\Sigma(V)),
$$

is the isomorphism $\eta_{V}=\left(\hat{\mathrm{e}}_{+}, \hat{\mathrm{e}}_{-}\right): V \rightarrow \Delta(\Sigma(V))=\left(\hat{U}^{+}, \hat{U}^{-}\right)$.

(d) For all $\mathscr{G}=\left(G, U^{+}, U^{-}\right) \in \mathbf{1}$-grp, the co-unit of the adjunction,

$$
\varepsilon_{\mathscr{G}}=\beta\left(\operatorname{Id}_{\Delta(\mathscr{G})}\right): \Sigma(\Delta(\mathscr{G})) \rightarrow \mathscr{G},
$$

is a covering, uniquely determined by $\varepsilon \mathscr{G}\left(\hat{\mathrm{e}}_{\sigma}(x)\right)=x$ for all $x \in U^{\sigma}$. It has the following universal property: for every covering $\varphi: \tilde{\mathscr{G}} \rightarrow \mathscr{G}$ of $\mathscr{G}$ there exists a unique covering $\psi: \Sigma(\Delta(\mathscr{G})) \rightarrow \tilde{\mathscr{G}}$ such that $\varphi \circ \psi=\varepsilon \mathscr{G}$.

Proof. (a) We verify the axioms (R0) - (R2) of a rank one group in 2.1. Axiom (R0) is trivially satisfied because $\hat{G}$ is by definition generated by $\hat{U}^{+}$and $\hat{U}^{-}$. For axiom (R1) assume $\hat{\mathrm{e}}_{+}(x)=\hat{\mathrm{e}}_{-}(y) \in \hat{U}^{+} \cap \hat{U}^{-}$. Applying $\hat{\pi}$, we see $\overline{\mathrm{e}}_{+}(x)=$ $\overline{\mathrm{e}}_{-}(y) \in \bar{U}^{+} \cap \bar{U}^{-}=\{1\}$ since $\overline{\mathscr{G}}$ is a rank one group, so $x=y=0$ by injectivity of $\overline{\mathrm{e}}_{\sigma}$.

To prove (R2), let $1 \neq \hat{\mathrm{e}}_{\sigma}(x) \in \hat{U}^{\sigma}$, so $0 \neq x \in V^{\sigma}$. We claim that

$$
\operatorname{Int}\left(\hat{\mathrm{e}}_{\sigma}(x)\right) \hat{U}^{-\sigma}=\operatorname{Int}\left(\hat{\mathrm{e}}_{-\sigma}(j(x))\right) U^{\sigma} .
$$

Indeed, by (3.1.3) and the fact that $R_{x}: V^{-\sigma} \rightarrow V^{\sigma}$ is bijective,

$$
\operatorname{Int}\left(\hat{\mathbf{w}}_{\sigma}(x)\right) \hat{U}^{-\sigma}=\hat{U}^{\sigma} .
$$

By (3.1.1) and since $\hat{\mathrm{e}}_{-\sigma}\left(x^{\vee}\right) \in \hat{U}^{-\sigma}$, this says

$$
\operatorname{Int}\left(\hat{\mathrm{e}}_{-\sigma}\left({ }^{\vee} x\right)\right) \operatorname{Int}\left(\hat{\mathrm{e}}_{\sigma}(x)\right) \hat{U}^{-\sigma}=\hat{U}^{\sigma} .
$$

Moreover, $\hat{\mathrm{e}}_{-\sigma}\left({ }^{\vee} x\right)^{-1}=\hat{\mathrm{e}}_{-\sigma}\left({ }^{\vee} x\right)=\hat{\mathrm{e}}_{-\sigma}(j(x))$ by (1.1.3). Hence conjugating (3.7.3) with $\hat{\mathrm{e}}_{-\sigma}(j(x))$ yields (3.7.2). Thus $\Sigma(V)$ is a rank one group, and (3.7.2) shows that the $j$-maps for $\hat{G}$ are

$$
j\left(\hat{\mathrm{e}}_{\sigma}(x)\right)=\hat{\mathrm{e}}_{-\sigma}(j(x)) .
$$

If $h: V \rightarrow W$ is a morphism of division pairs then $\operatorname{St}(h): \operatorname{St}(V) \rightarrow \operatorname{St}(W)$ is, by (3.1.4), a group homomorphism mapping $\hat{\mathrm{e}}_{\sigma}\left(V^{\sigma}\right)$ to $\hat{\mathrm{e}}_{\sigma}\left(W^{\sigma}\right)$. It follows from Lemma 3.6 and the fact that $h_{\sigma}$ is injective (see 1.1) that the restriction of St $(h)$ to $\hat{\mathrm{e}}_{\sigma}\left(V^{\sigma}\right)$ is injective as well. Hence $\mathrm{St}(h)$ is a morphism of rank one groups (as defined in 2.1). The functorial properties of $\Sigma$ are easily verified, and it is evident that it commutes with ( )op. 
(b) We define $\alpha$ and $\beta$ as follows. Let $\varphi: \Sigma(V)=\left(\hat{G}, \hat{U}^{+}, \hat{U}^{-}\right) \rightarrow \mathscr{G}=$ $\left(G, U^{+}, U^{-}\right)$be a morphism of rank one groups. Recall from 2.5 that $\Delta(\mathscr{G})=$ $\left(U^{+}, U^{-}\right)$with the $j$-maps defined in Lemma 2.2. Define $\alpha(\varphi)=h=\left(h_{+}, h_{-}\right)$: $V \rightarrow \Delta(\mathscr{G})$ by

$$
h_{\sigma}(x)=\varphi\left(\hat{\mathrm{e}}_{\sigma}(x)\right) \in U^{\sigma},
$$

i.e., by the commutative diagram

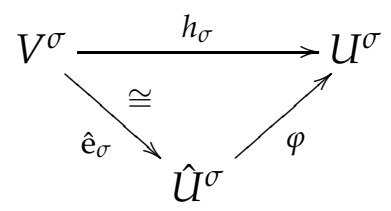

We must show that $h$ is a morphism of division pairs; i.e., that the $h_{\sigma}$ are injective group homomorphisms commuting with the $j$-maps, see (1.1.2). Now by (3.7.4) and (2.4.1),

$$
h_{-\sigma}(j(x))=\varphi\left(\hat{\mathrm{e}}_{-\sigma}(j(x))\right)=\varphi\left(j\left(\hat{\mathrm{e}}_{\sigma}(x)\right)=j\left(\varphi\left(\hat{\mathrm{e}}_{\sigma}(x)\right)=j\left(h_{\sigma}(x)\right) .\right.\right.
$$

This shows that $\alpha(\varphi): V \rightarrow \Delta(\mathscr{G})$ is a morphism of division pairs.

Conversely, let $h: V \rightarrow \Delta(\mathscr{G})=\left(U^{+}, U^{-}\right)$be a morphism of division pairs, and define first homomorphisms $\varphi_{\sigma}: \hat{U}^{\sigma} \rightarrow G$ by

$$
\varphi_{\sigma}\left(\hat{\mathrm{e}}_{\sigma}(x)\right):=h_{\sigma}(x) \in U^{\sigma} \quad\left(x \in V^{\sigma}\right) .
$$

Since $\hat{\mathrm{e}}_{\sigma}: V^{\sigma} \rightarrow \hat{U}^{\sigma}$ is an isomorphism, this makes sense. To see that the $\varphi_{\sigma}$ extend to a group homomorphism $\varphi: \hat{G} \rightarrow G$, we must show that they respect the defining relations (3.1.2) and (3.1.3) of $\operatorname{St}(V)$. For (3.1.2), this follows from the fact that $h_{\sigma}$ is a group homomorphism. For (3.1.3) we compute, using (1.2.3) and (2.7.1):

$$
\varphi_{\sigma}\left(\hat{\mathbf{e}}_{\sigma}\left(R_{x} y\right)\right)=h_{\sigma}\left(R_{x} y\right)=R_{h_{\sigma}(x)} h_{-\sigma}(y)=\operatorname{Int}\left(w_{h_{\sigma}(x)}\right) h_{-\sigma}(y) .
$$

By (1.1.6),

$$
w_{h_{\sigma}(x)}={ }^{\vee} h_{\sigma}(x) h_{\sigma}(x) h_{\sigma}(x)^{\vee}=h_{-\sigma}\left({ }^{\vee} x\right) h_{\sigma}(x) h_{-\sigma}\left(x^{\vee}\right) .
$$

This together with (3.7.7) shows that the maps $\varphi_{\sigma}$ preserve the relations (3.1.3) and hence extend to a unique group homomorphism $\varphi=\beta(h): \hat{G} \rightarrow G$. It maps $\hat{U}^{\sigma}$ injectively to $U^{\sigma}$, hence is a morphism of rank one groups.

It is easily seen that $\alpha$ and $\beta$ are inverses of each other and that they are natural. Details are left to the reader.

(c) Let $\mathscr{G}=\Sigma(V)$ and $\varphi=\mathrm{Id}$. Then by (3.7.5),

$$
\left(\eta_{V}\right)_{\sigma}(x)=\alpha(\varphi)_{\sigma}(x)=\hat{\mathrm{e}}_{\sigma}(x),
$$

so that $\eta_{V}=\left(\hat{\mathrm{e}}_{+}, \hat{\mathrm{e}}_{-}\right): V \rightarrow\left(\hat{U}^{+}, \hat{U}^{-}\right)$, and this is an isomorphism since the maps $\hat{\mathrm{e}}_{\sigma}: V^{\sigma} \rightarrow \hat{U}^{\sigma}$ are isomorphisms by Lemma 3.6.

(d) Let $h=\mathrm{Id}: \Delta(\mathscr{G}) \rightarrow \Delta(\mathscr{G})$ in (3.7.6). Then $\beta(\mathrm{Id})\left(\hat{\mathrm{e}}_{\sigma}(x)\right)=x \in U^{\sigma}$, hence $\pi:=\varepsilon_{\mathscr{G}}: \Sigma(\Delta(\mathscr{G})) \rightarrow \mathscr{G}$ is the homomorphism characterized by $\pi\left(\hat{\mathrm{e}}_{\sigma}(x)\right)=x$ 
for all $x \in U^{\sigma}$. This is obviously surjective on root groups, hence a covering by 2.8 .

Now let $\varphi: \tilde{\mathscr{G}} \rightarrow \mathscr{G}$ be any covering. By $2.8(\mathrm{ii}), h:=\Delta(\varphi): \Delta(\tilde{\mathscr{G}}) \rightarrow \Delta(\mathscr{G})$ is an isomorphism of division pairs, so $h^{-1}: \Delta(\mathscr{G}) \rightarrow \Delta(\tilde{\mathscr{G}})$ is a homomorphism of division pairs as well. The adjunction formula (3.7.1), with $\mathscr{G}$ replaced by $\tilde{\mathscr{G}}$ and $V=\Delta(\mathscr{G})$, yields inverse bijections

$$
\operatorname{Mor}_{1-\operatorname{grp}}(\Sigma(\Delta(\mathscr{G})), \tilde{\mathscr{G}}) \underset{\beta}{\stackrel{\alpha}{\rightleftarrows}} \operatorname{Mor}_{\operatorname{div}}(\Delta(\mathscr{G}), \Delta(\tilde{\mathscr{G}})) \text {. }
$$

Then $\psi:=\beta\left(h^{-1}\right): \Sigma(\Delta(\mathscr{G})) \rightarrow \tilde{\mathscr{G}}$ has the asserted properties.

Remark. In the terminology of [13, Definition 2.5], $\varepsilon_{\mathscr{G}}$ is a universal rank one extension of $\mathscr{G}$.

3.8 Corollary. Let $V$ be a division pair. Then the homomorphism $\hat{\pi}: \Sigma(V) \rightarrow \overline{\mathscr{G}}(V)$ of Lemma 3.6 is a covering of rank one groups, and $\operatorname{Ker}(\hat{\pi})=\mathscr{Z}(\operatorname{St}(V))$, so that $\mathrm{St}(V) / \mathscr{Z}(\operatorname{St}(V)) \cong \bar{G}$ as in 3.4 .

Proof. By Theorem 3.7(a) and 3.4, $\Sigma(V)$ and $\overline{\mathscr{G}}(V)$ are rank one groups. Since $\hat{\pi}$ is bijective on root groups by Lemma 3.6, it is a covering by 2.8(iii). By Lemma 3.3, $\bar{G}$ has trivial centre, so $\operatorname{Ker}(\hat{\pi})=\mathscr{Z}(\hat{G})$ follows from (2.8.1).

3.9 Corollary. Let $V$ be a Jordan division pair. Then $\operatorname{St}(V) / \mathscr{Z}(\operatorname{St}(V))$ is isomorphic to the projective elementary group $\mathrm{PE}(V)$ of $V$.

Proof. As noted in 3.2(a), the groups $\mathrm{St}(V)$ defined here and in [8] are the same. By [7, Corollary 6.6, Theorem 6.13], a Jordan division pair is unit-regular. Hence it follows from [8, Theorem 1.12] that $\mathrm{PE}(V) \cong \operatorname{St}(V) / \mathscr{Z}(\operatorname{St}(V))$.

3.10 Corollary. (a) Let $\mathscr{G}=\left(G, U^{+}, U^{-}\right)$and $\mathscr{G}^{\prime}=\left(G^{\prime}, U^{\prime+}, U^{\prime-}\right)$ be rank one groups with trivial centre and associated division pairs $V=\Delta(\mathscr{G})$ and $V^{\prime}=\Delta\left(\mathscr{G}^{\prime}\right)$, and let $h: V \rightarrow V^{\prime}$ be an isomorphism of division pairs. Then there exists a unique isomorphism $\psi: \mathscr{G} \rightarrow \mathscr{G}^{\prime}$ of rank one groups such that $\Delta(\psi)=h$.

(b) The functor $\Delta$ induces a bijection between the set of isomorphism classes of rank one groups with trivial centre and division pairs.

Proof. (a) Uniqueness of $\psi$ is clear because $G$ is generated by $U^{+}$and $U^{-}$. Since $\Sigma$ is a functor, $h$ induces an isomorphism

$$
\Sigma(h)=\varphi: \Sigma(V)=\left(\hat{G}, \hat{U}^{+}, \hat{U}^{-}\right) \stackrel{\cong}{\longrightarrow} \Sigma\left(V^{\prime}\right)=\left(\hat{G}^{\prime}, \hat{U}^{\prime+}, \hat{U}^{\prime-}\right) .
$$

By Theorem 3.7(d), $\varepsilon_{\mathscr{G}}: \Sigma(V) \rightarrow \mathscr{G}$ is a covering, and by $(2.8 .1), \operatorname{Ker}\left(\varepsilon_{\mathscr{G}}\right)=$ $\varepsilon_{\mathscr{G}}^{-1}(\mathscr{Z}(G))=\mathscr{Z}(\operatorname{St}(V))$, and the same holds for $\varepsilon_{\mathscr{G} \prime}$. Since $\varphi$ is a group isomorphism, it maps the respective centres isomorphically to each other, and hence 
induces an isomorphism $\psi: \mathscr{G} \rightarrow \mathscr{G}^{\prime}$ making the diagram

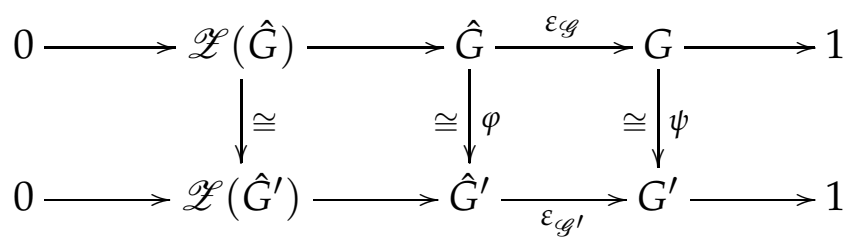

commutative. Thus for $x \in U^{\sigma}$, we have $\psi\left(\varepsilon_{\mathscr{G}}\left(\hat{\mathrm{e}}_{\sigma}(x)\right)\right)=\varepsilon_{\mathscr{G}}\left(\varphi\left(\hat{\mathrm{e}}_{\sigma}(x)\right)\right)$. By Theorem 3.7(d), $\varepsilon_{\mathscr{G}}\left(\hat{\mathrm{e}}_{\sigma}(x)\right)=x$, and by (3.1.4), $\varphi\left(\hat{\mathrm{e}}_{\sigma}(x)\right)=\hat{\mathrm{e}}_{\sigma}^{\prime}\left(h_{\sigma}(x)\right)$, so $\varepsilon_{\varphi_{G}}\left(\varphi\left(\hat{\mathrm{e}}_{\sigma}(x)\right)\right)$ $=\varepsilon \mathscr{G}^{\prime}\left(\hat{\mathrm{e}}_{\sigma}^{\prime}\left(h_{\sigma}(x)\right)\right)=h_{\sigma}(x)$, which implies $\psi(x)=h_{\sigma}(x)$ and therefore $\Delta(\psi)=h$, as desired.

(b) Let $\bar{\Delta}$ be the map induced by $\Delta$ on the respective isomorphism classes. We saw in 3.4 that for every $V \in \operatorname{div}$ there exists a centre-free rank one group $\overline{\mathscr{G}}$ with $\Delta(\overline{\mathscr{G}}) \cong V$, so $\bar{\Delta}$ is surjective. Injectivity of $\bar{\Delta}$ follows from (a).

\section{Identities in rank one groups and division pairs}

4.1 Notation. In this section, we derive identities in rank one groups and division pairs. The following notations and conventions will be in force throughout. Consider a rank one group $\mathscr{G}=\left(G, U^{+}, U^{-}\right)$, a division pair $V=\left(V^{+}, V^{-}\right)$and an isomorphism e: $V \rightarrow \Delta(\mathscr{G})$. Thus the $\mathrm{e}_{\sigma}: V^{\sigma} \rightarrow U^{\sigma}$ are group isomorphisms satisfying

$$
j\left(\mathrm{e}_{\sigma}(x)\right)=\mathrm{e}_{-\sigma}(j(x))
$$

and therefore, by the definition of the $j$-maps in $\Delta(\mathscr{G})$,

$$
\operatorname{Int}\left(\mathrm{e}_{\sigma}(x)\right) U^{-\sigma}=\operatorname{Int}\left(\mathrm{e}_{-\sigma}(j(x))\right) U^{\sigma}
$$

for all $x \in \dot{V}^{\sigma}, \sigma \in\{+,-\}$. These are just the formulas (3.7.4) and (3.7.2) in the present notation. Using the action of $G$ on $\Xi$ as in (2.2.4), (4.1.2) is equivalent to

$$
\mathrm{e}_{\sigma}(x) \cdot o^{-\sigma}=\mathrm{e}_{-\sigma}(j(x)) \cdot o^{\sigma} .
$$

The Weyl element determined by $x$ is

$$
\mathrm{w}_{\sigma}(x)=\mathrm{e}_{-\sigma}\left({ }^{\vee} x\right) \mathrm{e}_{\sigma}(x) \mathrm{e}_{-\sigma}\left(x^{\vee}\right) \in G .
$$

In the notation of Lemma 2.6,

$$
\mathrm{w}_{\sigma}(x)=w_{\mathbf{e}_{\sigma}(x)} .
$$

Hence by (2.6.2), Int $\mathrm{w}_{\sigma}(x)$ interchanges $U^{+}$and $U^{-}$, and by (2.7.1) we have

$$
\operatorname{Int}\left(\mathrm{w}_{\sigma}(x)\right) \mathrm{e}_{-\sigma}(y)=R_{\mathrm{e}_{\sigma}(x)} \mathrm{e}_{-\sigma}(y)=\mathrm{e}_{\sigma}\left(R_{x} y\right) .
$$

By Theorem 3.7, every division pair $V$ is isomorphic to $\Delta(\mathscr{G})$ for some rank one group. Hence, any identity for $V$ derived in the present setting will be valid in an arbitrary division pair.

It would of course be possible to dispense with the isomorphisms $\mathrm{e}_{\sigma}$ and work directly with the division pair $\Delta(\mathscr{G})=\left(U^{+}, U^{-}\right)$; in other words, put $V^{\sigma}=U^{\sigma}$ and $\mathrm{e}_{\sigma}=\mathrm{Id}$. This would make for shorter but also for more error-prone and less readable formulas. Therefore, we use the lengthier notation. The $\mathrm{e}_{\sigma}$ may be considered as formal exponential maps. 
4.2 Lemma. For $x \in \dot{V}^{\sigma}, z \in V^{\sigma}$ and $y \in V^{-\sigma}$, we have the formulas

$$
\begin{aligned}
R_{x}\left(x^{\vee}\right) & ={ }^{\vee}{ }^{\vee} x, \quad R_{-x}\left({ }^{\vee} x\right)=x^{\vee \vee}, \\
\mathrm{w}_{\sigma}(x) & =\mathrm{w}_{-\sigma}\left({ }^{\vee} x\right)=\mathrm{w}_{-\sigma}\left(x^{\vee}\right), \\
\mathrm{w}_{\sigma}(x)^{-1} & =\mathrm{w}_{\sigma}(-x)=\mathrm{w}_{-\sigma}(j(x)), \\
\operatorname{Int}\left(\mathrm{w}_{\sigma}(x)\right) \mathrm{e}_{\sigma}(z) & =\mathrm{e}_{-\sigma}\left(R\left(x^{\vee}\right) z\right), \\
R_{x}^{-1} & =R_{j(x)}, \\
R\left({ }^{\vee} x\right) & =R\left(x^{\vee}\right)=R_{-x}^{-1} .
\end{aligned}
$$

Proof. Let $y=-x^{\vee}$, so that $x^{\vee}+y=0$. Since $R_{x}$ is a group homomorphism, (1.2.1) implies

$$
R_{x}\left(x^{\vee}\right)=R_{x}(-y)=-R_{x} y=-j\left({ }^{\vee} x\right)={ }^{\vee}{ }^{\vee} x .
$$

This proves the first formula of (4.2.1), and the second follows by replacing $x$ with $-x$ :

$$
R_{-x}\left({ }^{\vee} x\right)=-R_{-x} j(x)=-R_{-x}(-x)^{\vee}=-{ }^{\vee \vee}(-x)=x^{\vee \vee} .
$$

By (4.1.6), $\operatorname{Int}\left(\mathrm{w}_{\sigma}(x)\right) \mathrm{e}_{-\sigma}\left(x^{\vee}\right)=\mathrm{e}_{\sigma}\left(R_{x} x^{\vee}\right)=\mathrm{e}_{\sigma}\left({ }^{\vee} x\right)$, and hence

$$
w:=\left[\operatorname{Int}\left(\mathrm{w}_{\sigma}(x)\right) \mathrm{e}_{-\sigma}\left(x^{\vee}\right)\right] \mathrm{e}_{-\sigma}\left({ }^{\vee} x\right) \mathrm{e}_{\sigma}(x)=\mathrm{e}_{\sigma}\left({ }^{\vee} \vee x\right) \mathrm{e}_{-\sigma}\left({ }^{\vee} x\right) \mathrm{e}_{\sigma}(x)=\mathrm{w}_{-\sigma}\left({ }^{\vee} x\right) .
$$

On the other hand, computing $w$ differently,

$$
w=\mathrm{w}_{\sigma}(x) \mathrm{e}_{-\sigma}\left(x^{\vee}\right) \mathrm{e}_{-\sigma}\left(x^{\vee}\right)^{-1} \mathrm{e}_{\sigma}(x)^{-1} \mathrm{e}_{-\sigma}\left({ }^{\vee} x\right)^{-1} \mathrm{e}_{-\sigma}\left({ }^{\vee} x\right) \mathrm{e}_{\sigma}(x)=\mathrm{w}_{\sigma}(x) .
$$

Hence we have the first equality of (4.2.2). The equality $\mathrm{w}_{\sigma}(x)=\mathrm{w}_{-\sigma}\left(x^{\vee}\right)$ follows by replacing $\sigma$ with $-\sigma$ and $x$ with $x^{\vee}$, which yields $\mathrm{w}_{-\sigma}\left(x^{\vee}\right)=\mathrm{w}_{\sigma}\left({ }^{\vee}\left(x^{\vee}\right)\right)=$ $\mathrm{w}_{\sigma}(x)$ by (1.1.4).

The first formula of (4.2.3) was noted before in (2.6.1), it follows immediately from (4.1.4) and (1.1.5). For the second, we have

$$
\mathrm{w}_{-\sigma}(j(x))=\mathrm{w}_{-\sigma}\left(-{ }^{\vee} x\right)=\mathrm{w}_{-\sigma}\left({ }^{\vee} x\right)^{-1}=\mathrm{w}_{\sigma}(x)^{-1} .
$$

For (4.2.4), we use (4.2.2) and (4.1.6), with $\sigma$ replaced by $-\sigma$ :

$$
\operatorname{Int}\left(\mathrm{w}_{\sigma}(x)\right) \mathrm{e}_{\sigma}(z)=\operatorname{Int}\left(\mathrm{w}_{-\sigma}\left(x^{\vee}\right)\right) \mathrm{e}_{\sigma}(z)=\mathrm{e}_{-\sigma}\left(R\left(x^{\vee}\right) z\right) .
$$

Combining $\mathrm{w}_{\sigma}(x)^{-1}=\mathrm{w}_{-\sigma}(j(x))$ with (4.1.6) shows (4.2.5). From (4.2.2) and (4.1.6) (with $\sigma$ replaced by $-\sigma$ ) we obtain $R\left({ }^{\vee} x\right)=R\left(x^{\vee}\right): V^{-\sigma} \rightarrow V^{\sigma}$, and by (4.2.5), $R\left(x^{\vee}\right)=R(j(-x))=R_{-x}^{-1}$. This proves (4.2.6).

4.3 Proposition. Let $\mathscr{G}^{\mathrm{op}}=\left(G, U^{-}, U^{+}\right)$and $V^{\mathrm{op}}=\left(V^{-}, V^{+}\right)$be the opposites as in 2.1 and 1.1. We keep the conventions of 4.1 and let $x \in \dot{V}^{\sigma}$.

(a) Let $\varphi=\operatorname{Int}_{\sigma}(x): G \rightarrow G$. Then $\varphi: \mathscr{G}$ op $\rightarrow \mathscr{G}$ is an isomorphism of rank one groups. For all $y \in \dot{V}^{-\sigma}$ we have

$$
\mathrm{w}_{\sigma}(x) \mathrm{w}_{-\sigma}(y) \mathrm{w}_{\sigma}(x)^{-1}=\mathrm{w}_{\sigma}\left(R_{x} y\right) .
$$


(b) Let $h_{\sigma}=R_{x}: V^{-\sigma} \rightarrow V^{\sigma}$ and $h_{-\sigma}=R\left(x^{\vee}\right): V^{\sigma} \rightarrow V^{-\sigma}$. Then $h=$ $\left(h_{+}, h_{-}\right): V^{\mathrm{op}} \rightarrow V$ is an isomorphism of division pairs. For all $z \in \dot{V}^{\sigma}$ and $y \in \dot{V}^{-\sigma}$, the following formulas hold:

$$
\begin{aligned}
R\left(R_{x} y\right) & =R_{x} R_{y} R_{-x}, & & \\
R_{x} \circ j & =j \circ R\left(x^{\vee}\right), & & \\
\left(R_{x} y\right)^{\vee} & =R\left(x^{\vee}\right) y^{\vee}, & & \vee \\
R_{x}\left(y^{\vee} y\right)=R\left({ }^{\vee} x\right)^{\vee} y, & =\left(R_{x} y\right)^{\vee \vee}, & & R_{x}\left({ }^{\vee} y\right)={ }^{\vee}\left(R_{x} y\right) .
\end{aligned}
$$

Proof. (a) Since $\varphi$ is an automorphism of $G$ interchanging $U^{+}$and $U^{-}$, it is an isomorphism from $\mathscr{G}$ op to $\mathscr{G}$. Let $v=\mathrm{e}_{-\sigma}(y) \in \dot{U}^{-\sigma}$. Then by (2.6.4), $\varphi\left(w_{v}\right)=w_{\varphi(v)}$, and by (4.1.5), $w_{v}=w_{\mathrm{e}_{-\sigma}(y)}=\mathrm{w}_{-\sigma}(y)$. Hence by (4.1.6), $\varphi(v)=\operatorname{Int}\left(\mathrm{w}_{\sigma}(x)\right) \mathrm{e}_{-\sigma}(y)$ $=\mathrm{e}_{\sigma}\left(R_{x} y\right)$ and therefore

$$
\operatorname{Int}\left(\mathrm{w}_{\sigma}(x)\right) \mathrm{w}_{-\sigma}(y)=\varphi\left(w_{v}\right)=w_{\varphi(v)}=w_{\mathrm{e}_{\sigma}\left(R_{x} y\right)}=\mathrm{w}_{\sigma}\left(R_{x} y\right),
$$

proving (4.3.1).

(b) By $2.5, \Delta$ is a functor commuting with ( ) ${ }^{\text {op }}$. Hence $\varphi$ induces an isomorphism $\Delta(\varphi): \Delta(\mathscr{G})^{\mathrm{op}} \rightarrow \Delta(\mathscr{G})$ and, by intertwining with the isomorphism $\mathrm{e}: V \rightarrow \Delta(\mathscr{G})$, an isomorphism $h=\mathrm{e}^{-1} \circ \Delta(\varphi) \circ \mathrm{e}^{\mathrm{op}}: V^{\mathrm{op}} \rightarrow V$. It follows from (4.1.6) that

$$
\Delta(\varphi) \mathrm{e}_{-\sigma}(y)=\mathrm{e}_{\sigma}\left(R_{x} y\right),
$$

so $h_{\sigma}(y)=R_{x} y$. Moreover, $\Delta(\varphi) \mathrm{e}_{\sigma}(z)=\operatorname{Int}\left(\mathrm{w}_{\sigma}(x)\right) \mathrm{e}_{\sigma}(z)=\mathrm{e}_{-\sigma}\left(R\left(x^{\vee}\right) z\right)$ by (4.2.4), which shows that $h_{-\sigma}(z)=R\left(x^{\vee}\right) z$, so $h=\left(h_{+}, h_{-}\right)$is an isomorphism of division pairs.

Formula (4.3.2) (the "fundamental formula") can be derived from (4.3.1) or from the fact that $h$ is an isomorphism, as follows. By (1.2.3), $h_{\sigma} R_{y}=R_{h_{\sigma}(y)} h_{-\sigma}$ for all $y \in\left(V^{\mathrm{op}}\right)^{\sigma}=V^{-\sigma}$. Hence $R_{x} R_{y}=R\left(R_{x} y\right) R\left(x^{\vee}\right)=R\left(R_{x} y\right) R_{-x}^{-1}$ (by (4.2.6)).

Since $h$ is an isomorphism of division pairs, it commutes with the maps ()$^{\vee}$ and $\vee($ ) by (1.1.6). This implies

$$
R_{x} j(y)=h_{\sigma}(j(y))=j\left(h_{-\sigma}(y)\right)=j\left(R\left(x^{\vee}\right)(y)\right)
$$

for all $x \in \dot{V}^{\sigma}, y \in \dot{V}^{-\sigma}$, which is equivalent to (4.3.3). The remaining formulas follow from this and the fact that $R_{x}$ is a group homomorphism, hence commutes with the group inverse.

4.4 The quasi-inverse. We now extend to division pairs some of the properties of the quasi-inverse in Jordan pairs [5, §3]. Let $V$ be a division pair. A pair $(x, y) \in$ $V^{\sigma} \times V^{-\sigma}$ is called quasi-invertible if $x=0$ or $y=0$ or $x \neq{ }^{\vee} y$; equivalently, by applying ()$^{\vee}$, that $y \neq x^{\vee}$. Thus the not quasi-invertible pairs are the pairs $\left(x, x^{\vee}\right), x \in \dot{V}^{\sigma}$, the graph of the map $x \mapsto x^{\vee}$. For a Jordan division pair this definition is consistent with the usual notion of quasi-invertibility [9, Lemma 1.9]. 
Given a quasi-invertible pair $(x, y) \in V^{\sigma} \times V^{-\sigma}$, we define the left and the right quasi-inverse of $(x, y)$ by

$$
\begin{aligned}
& \ell_{\sigma}(x, y)={ }^{x} y=\left\{\begin{array}{ll}
\left({ }^{\vee} y-x\right)^{\vee} & \text { if } y \neq 0 \\
0 & \text { if } y=0
\end{array}\right\} \in V^{-\sigma},
\end{aligned}
$$

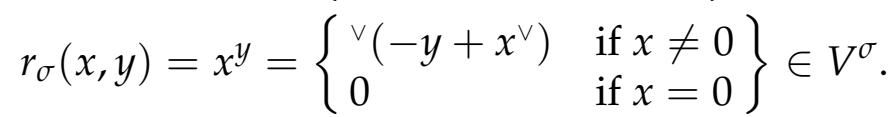

Clearly, any pair $(x, 0)$ and $(0, y)$ is quasi-invertible, so the main interest is in case $x \neq 0$ and $y \neq 0$.

4.5 Lemma. (a) Let $h: V \rightarrow W$ be a homomorphism of division pairs. Then $(x, y)$ is quasi-invertible if and only if $\left(h_{\sigma}(x), h_{-\sigma}(y)\right)$ is quasi-invertible, in which case

$$
h_{-\sigma}\left(\ell_{\sigma}(x, y)\right)=\ell_{\sigma}\left(h_{\sigma}(x), h_{-\sigma}(y)\right), \quad h_{\sigma}\left(r_{\sigma}(x, y)\right)=r_{\sigma}\left(h_{\sigma}(x), h_{-\sigma}(y)\right) .
$$

(b) $(x, y)$ is quasi-invertible if and only if $(-y,-x)$ is quasi-invertible, and then

$$
-\left(x^{y}\right)=(-y)(-x), \quad-\left({ }^{x} y\right)=(-y)^{(-x)} .
$$

Proof. (a) This follows immediately from the fact that the $h_{\sigma}$ are injective group homomorphisms commuting with the maps ${ }^{\vee}()$ and ()$^{\vee}$.

(b) There is no harm in assuming $x \neq 0$ and $y \neq 0$. Then by (1.1.5),

$$
(-y,-x) \text { quasi-invertible } \Longleftrightarrow-y \neq^{\vee}(-x)=-x^{\vee} \Longleftrightarrow y \neq x^{\vee}
$$

Moreover,

$$
\begin{aligned}
\ell_{-\sigma}(-y,-x) & =\left({ }^{\vee}(-x)-(-y)\right)^{\vee}=\left(-x^{\vee}+y\right)^{\vee} \\
& =-{ }^{\vee}\left(-\left(-x^{\vee}+y\right)\right)=-{ }^{\vee}\left(-y+x^{\vee}\right)=-r_{\sigma}(x, y),
\end{aligned}
$$

and the second formula follows from this by the substitution $x \mapsto-y$ and $y \mapsto-x$.

The characterization of quasi-invertibility in the next result is similar to the one for Jordan pairs in [6, Theorem 4.1], see also [4, Lemma 3] and [7, Theorem 1.4].

4.6 Proposition. Recall the subsets $\Omega^{\sigma}=U^{-\sigma} H U^{\sigma}$ of $G$ from (2.2.7) and let $(x, y) \in V^{\sigma} \times V^{-\sigma}$. Then

$$
(x, y) \text { is quasi-invertible } \Longleftrightarrow \mathrm{e}_{\sigma}(x) \mathrm{e}_{-\sigma}(y) \in \Omega^{\sigma} .
$$

In this case,

$$
\mathrm{e}_{\sigma}(x) \mathrm{e}_{-\sigma}(y)=\mathrm{e}_{-\sigma}\left({ }^{x} y\right) \mathrm{b}_{\sigma}(x, y) \mathrm{e}_{\sigma}\left(x^{y}\right)
$$

where $\mathrm{b}_{\sigma}(x, y) \in H$ is given by

$$
\mathrm{b}_{\sigma}(x, y)=\mathrm{w}_{\sigma}\left(x-{ }^{\vee} y\right) \mathrm{w}_{-\sigma}(y)=\mathrm{w}_{\sigma}(x) \mathrm{w}_{-\sigma}\left(-x^{\vee}+y\right)
$$


and satisfies

$$
\mathrm{b}_{\sigma}(x, y)^{-1}=\mathrm{b}_{-\sigma}(-y,-x)
$$

Proof. If either $x=0$ or $y=0$ this is trivial (with $\mathrm{b}_{\sigma}(x, 0)=\mathrm{b}_{\sigma}(0, y)=1$ ), so we assume $x \neq 0$ and $y \neq 0$. To prove (4.6.1), we use Lemma 2.3(e) and (4.1.3):

$$
\begin{aligned}
\mathrm{e}_{\sigma}(x) \mathrm{e}_{-\sigma}(y) \cdot o^{\sigma} & =\mathrm{e}_{\sigma}(x) \mathrm{e}_{\sigma}(j(y)) \cdot o^{-\sigma}=\mathrm{e}_{\sigma}(x+j(y)) \cdot o^{-\sigma} \\
& =\mathrm{e}_{\sigma}\left(x-{ }^{\vee} y\right) \cdot o^{-\sigma} .
\end{aligned}
$$

Since $U^{\sigma}$ acts freely on $\Xi-\left\{o^{\sigma}\right\}$ by Lemma 2.3(a), this shows

$$
\mathrm{e}_{\sigma}(x) \mathrm{e}_{-\sigma}(y) \in \Omega^{\sigma} \Longleftrightarrow x-{ }^{\vee} y \neq 0 \quad \Longleftrightarrow \quad(x, y) \text { quasi-invertible. }
$$

Now assume $(x, y)$ quasi-invertible. Then (4.6.5) and (4.1.3) imply

$$
\mathrm{e}_{\sigma}(x) \mathrm{e}_{-\sigma}(y) \cdot o^{\sigma}=\mathrm{e}_{-\sigma}\left(j\left(x-{ }^{\vee} y\right)\right) \cdot o^{\sigma}=\mathrm{e}_{-\sigma}\left(\left({ }^{\vee} y-x\right)^{\vee}\right) \cdot o^{\sigma}=\mathrm{e}_{-\sigma}\left({ }^{x} y\right) \cdot o^{\sigma},
$$

so $\mathrm{e}_{\sigma}(x) \mathrm{e}_{-\sigma}(y) \in \mathrm{e}_{-\sigma}\left({ }^{x} y\right) B^{\sigma}$ since $B^{\sigma}=H U^{\sigma}$ is the isotropy group of $o^{\sigma}$. It follows that

$$
\mathrm{e}_{\sigma}(x) \mathrm{e}_{-\sigma}(y)=\mathrm{e}_{-\sigma}\left({ }^{x} y\right) \mathrm{b}_{\sigma}(x, y) u
$$

for uniquely determined $\mathrm{b}_{\sigma}(x, y)=h \in H$ and $u \in U^{\sigma}$ and it remains to determine $h$ and $u$. Inverting (4.6.6) yields

$$
\mathrm{e}_{-\sigma}(-y) \mathrm{e}_{\sigma}(-x)=u^{-1} \mathrm{~b}_{\sigma}(x, y)^{-1} \mathrm{e}_{-\sigma}\left(-\left({ }^{x} y\right)\right)
$$

On the other hand, by Lemma $4.5(\mathrm{~b}),(-y,-x)$ is quasi-invertible along with $(x, y)$ and the quasi-inverses are related by (4.5.2). Hence (4.6.6), with $\sigma, x, y$ replaced by $-\sigma,-y,-x$ yields

$$
\mathrm{e}_{-\sigma}(-y) \mathrm{e}_{\sigma}(-x)=\mathrm{e}_{\sigma}\left({ }^{(-y)}(-x)\right) \mathrm{b}_{\sigma}(-y,-x) v=\mathrm{e}_{\sigma}\left(-\left(x^{y}\right)\right) \mathrm{b}_{-\sigma}(-y,-x) v
$$

for unique $\mathrm{b}_{-\sigma}(-y,-x) \in H$ and $v \in U^{-\sigma}$. Comparing (4.6.7) and (4.6.8) shows, by Lemma $2.3(\mathrm{e})$, that $\mathrm{b}_{\sigma}(x, y)^{-1}=\mathrm{b}_{-\sigma}(-y,-x)$ and $u=\mathrm{e}_{\sigma}\left(x^{y}\right)$, so we have (4.6.2) and (4.6.4).

It remains to prove (4.6.3). We put $z=x-{ }^{\vee} y \in \dot{V}^{\sigma}$ and compute $\mathrm{w}_{\sigma}(z) \mathrm{w}_{-\sigma}(y)$. Observe that ${ }^{x} y=\left({ }^{\vee} y-x\right)^{\vee}=(-z)^{\vee}=-{ }^{\vee} z$ so that ${ }^{\vee} z+{ }^{x} y=0$. Hence, using (4.2.2) on the first factor, formula (4.6.2) and $z+{ }^{\vee} y=x$,

$$
\begin{aligned}
\mathrm{w}_{\sigma}(z) \mathrm{w}_{-\sigma}(y) & =\mathrm{w}_{-\sigma}\left({ }^{\vee} z\right) \mathrm{w}_{-\sigma}(y) \\
& =\mathrm{e}_{\sigma}\left({ }^{\vee \vee} z\right) \mathrm{e}_{-\sigma}\left({ }^{\vee} z\right) \mathrm{e}_{\sigma}\left(z+{ }^{\vee} y\right) \mathrm{e}_{-\sigma}(y) \mathrm{e}_{\sigma}\left(y^{\vee}\right) \\
& =\mathrm{e}_{\sigma}\left({ }^{\vee \vee} z\right) \mathrm{e}_{-\sigma}\left({ }^{\vee} z\right)\left[\mathrm{e}_{\sigma}(x) \mathrm{e}_{-\sigma}(y)\right] \mathrm{e}_{\sigma}\left(y^{\vee}\right) \\
& =\mathrm{e}_{\sigma}\left({ }^{\vee \vee} z\right) \mathrm{e}_{-\sigma}\left({ }^{\vee} z\right)\left[\mathrm{e}_{-\sigma}\left({ }^{x} y\right) \mathrm{b}_{\sigma}(x, y) \mathrm{e}_{\sigma}\left(x^{y}\right)\right] \mathrm{e}_{\sigma}\left(y^{\vee}\right) \\
& =\mathrm{e}_{\sigma}\left({ }^{\vee \vee} z\right) \mathrm{e}_{-\sigma}\left({ }^{\vee} z+{ }^{x} y\right) \mathrm{b}_{\sigma}(x, y) \mathrm{e}_{\sigma}\left(x^{y}+y^{\vee}\right) \\
& =\mathrm{e}_{\sigma}\left({ }^{\vee \vee} z\right) \mathrm{b}_{\sigma}(x, y) \mathrm{e}_{\sigma}\left(x^{y}+y^{\vee}\right) \in U^{\sigma} H U^{\sigma} .
\end{aligned}
$$

Now Int $\mathrm{w}_{\sigma}(z)$ and Int $\mathrm{w}_{-\sigma}(y)$ both interchange $U^{+}$and $U^{-}$, so their product normalizes $U^{+}$and $U^{-}$and therefore belongs to $H$. Since $U^{\sigma} \cap H \subset U^{\sigma} \cap B^{-\sigma}=$ $\{1\}$ by Lemma 2.2(a), it follows that $\mathrm{w}_{\sigma}(z) \mathrm{w}_{-\sigma}(y)=\mathrm{b}_{\sigma}(x, y)$. 
By (4.6.4) and (4.2.3),

$$
\begin{aligned}
\mathrm{b}_{-\sigma}(-y,-x) & =\mathrm{b}_{\sigma}(x, y)^{-1}=\mathrm{w}_{-\sigma}(y)^{-1} \mathrm{w}_{\sigma}\left(x-{ }^{\vee} y\right)^{-1} \\
& =\mathrm{w}_{-\sigma}(-y) \mathrm{w}_{\sigma}\left({ }^{\vee} y-x\right) .
\end{aligned}
$$

Now the substitution $\sigma \mapsto-\sigma, x \mapsto-y$ and $y \mapsto-x$ yields the missing relation $\mathrm{b}_{\sigma}(x, y)=\mathrm{w}_{\sigma}(x) \mathrm{w}_{-\sigma}\left(-x^{\vee}+y\right)$.

4.7 Corollary. For quasi-invertible $(x, y) \in \dot{V}^{\sigma} \times \dot{V}^{-\sigma}$, the following formulas hold:

$$
\begin{aligned}
\mathrm{w}_{\sigma}(x) & =\mathrm{b}_{\sigma}(x, y) \mathrm{w}_{\sigma}\left(x^{y}\right), \\
\mathrm{w}_{\sigma}(-x) & =\mathrm{w}_{\sigma}((-y)(-x)) \mathrm{b}_{-\sigma}(-y,-x), \\
\mathrm{b}_{\sigma}(x, y) \mathrm{w}_{\sigma}(-x) & =\mathrm{w}_{\sigma}\left((-x)^{\vee \vee}+R_{x} y\right), \\
\mathrm{w}_{-\sigma}(-y) \mathrm{b}_{\sigma}(x, y) & =\mathrm{w}_{-\sigma}\left(R_{-y} x+{ }^{\vee \vee}(-y)\right) .
\end{aligned}
$$

Proof. For (4.7.1), we use the second form of $b_{\sigma}(x, y)$ from (4.6.3) as well as the formulas of Lemma 4.2:

$$
\begin{aligned}
\mathrm{b}_{\sigma}(x, y) \mathrm{w}_{\sigma}\left(x^{y}\right) & =\mathrm{w}_{\sigma}(x) \mathrm{w}_{-\sigma}\left(-x^{\vee}+y\right) \mathrm{w}_{\sigma}\left({ }^{\vee}\left(-y+x^{\vee}\right)\right) \\
& =\mathrm{w}_{\sigma}(x) \mathrm{w}_{-\sigma}\left(-x^{\vee}+y\right) \mathrm{w}_{-\sigma}\left(-y+x^{\vee}\right) \\
& =\mathrm{w}_{\sigma}(x) \mathrm{w}_{-\sigma}\left(-x^{\vee}+y\right) \mathrm{w}_{-\sigma}\left(-x^{\vee}+y\right)^{-1}=\mathrm{w}_{\sigma}(x) .
\end{aligned}
$$

Now (4.7.2) follows by inverting (4.7.1) and using (4.2.3) and (4.6.4):

$$
\mathrm{w}_{\sigma}(-x)=\mathrm{w}_{\sigma}\left(-\left(x^{y}\right)\right) \mathrm{b}_{\sigma}(x, y)^{-1}=\mathrm{w}_{\sigma}\left({ }^{(-y)}(-x)\right) \mathrm{b}_{-\sigma}(-y,-x) .
$$

Next, by (4.6.3) and (4.3.1),

$$
\begin{aligned}
\mathrm{b}_{\sigma}(x, y) \mathrm{w}_{\sigma}(-x) & =\mathrm{w}_{\sigma}(x) \mathrm{w}_{-\sigma}\left(-x^{\vee}+y\right) \mathrm{w}_{\sigma}(-x) \\
& =\mathrm{w}_{\sigma}\left(R_{x}\left(-x^{\vee}+y\right)\right)=\mathrm{w}_{\sigma}\left(-R_{x}\left(x^{\vee}\right)+R_{x} y\right),
\end{aligned}
$$

since $R_{x}$ is a group homomorphism. Now $R_{x} x^{\vee}={ }^{\vee} \vee x$ by (4.2.1), so $-R_{x}\left(x^{\vee}\right)=$ $-{ }^{\vee} x=(-x)^{\vee \vee}$ by (1.1.5), and (4.7.3) follows. Finally, (4.7.4) follows from (4.7.3) by inverting, using (4.6.4) and the substitution $x \mapsto-y, y \mapsto-x$.

4.8 Relation with the identities of De Medts and Segev [2]. We now show that the identities of [2, Theorem 1.1] are consequences of our identities (4.3.1) and (4.6.3). De Medts and Segev work in the little projective group of a Moufang set, that is, a rank one group with trivial centre by Lemma 3.3 and Corollary 3.10, whereas our identities hold in any rank one group. For the convenience of the reader, we first review the construction of Moufang sets from division pairs in [9] and from pairs $(U, \tau)$ in [3].

Let $V$ be a pre-division pair, let $\Gamma=\left\{(x, y) \in \dot{V}^{+} \times \dot{V}^{-}: y=j_{+}(x)\right\}$ be the graph of $j_{+}$, and let $\pi_{\sigma}: \Gamma \rightarrow \dot{V}^{\sigma} \subset V^{\sigma}$ be the projections followed by the inclusions. We put

$$
\mathbb{X}(V)=V^{+} \sqcup_{\Gamma} V^{-}
$$


the amalgamated sum (pushout) of $V^{+}$and $V^{-}$over $\Gamma$, with canonical maps $\iota_{\sigma}: V^{\sigma} \rightarrow \mathbb{X}(V)$. Thus $\iota_{+} \circ \pi_{+}=\iota_{-} \circ \pi_{-}$, and $\left(\mathbb{X}(V), \iota_{+}, \iota_{-}\right)$has the following property: for every pair of maps $\beta_{+}: V^{+} \rightarrow X$ and $\beta_{-}: V^{-} \rightarrow X$ into some set $X$ satisfying $\beta_{+} \circ \pi_{+}=\beta_{-} \circ \pi_{-}$, there exists a unique map $\beta: \mathbb{X}(V) \rightarrow X$ such that $\beta \circ \iota_{\sigma}=\beta_{\sigma}$ :

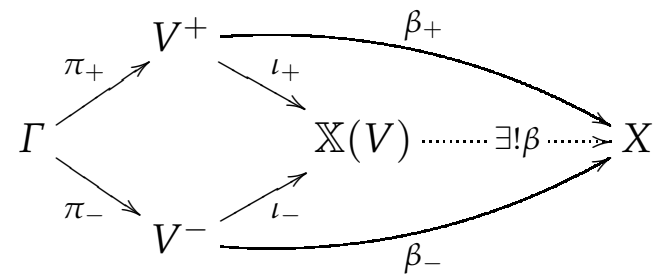

The triple $\left(\mathbb{X}(V), \iota_{+}, \iota_{-}\right)$is uniquely determined up to unique isomorphism by this property. The construction is well-known: $\mathbb{X}(V)$ is the quotient of the disjoint union of $V^{+}$and $V^{-}$by the equivalence relation which is trivial on $V^{\sigma}$ and identifies $x \in \dot{V}^{+}$with $j_{+}(x) \in \dot{V}^{-}$. The maps $\iota_{\sigma}$ are then the inclusions of $V^{\sigma}$ into the disjoint union followed by the canonical map into the quotient.

We specialize this to the following situation. Let $U$ be a group, not necessarily abelian but written additively, and let $\tau: \dot{U} \rightarrow \dot{U}$ be a bijection. Then [9, Section 4$]$ the pair of groups $V=(U, U)$ with the maps

$$
j_{+}=\tau^{-1}, \quad j_{-}=\tau
$$

is a pre-division pair. Let $X=U \cup\{\infty\}$ where $\infty$ is a new symbol, and define $\beta_{\sigma}: V^{\sigma}=U \rightarrow X$ by $\beta_{+}(x)=x, \beta_{-}(y)=\tau(y)$ for $y \neq 0$ while $\beta_{-}(0)=\infty$. Then it follows from (4.8.1) that there is a bijection $\beta: \mathbb{X}(V) \rightarrow X$ given by

$$
\beta\left(\iota_{+}(x)\right)=x, \quad \beta\left(\iota_{-}(y)\right)=\left\{\begin{array}{ll}
\infty & \text { if } y=0 \\
\tau(y) & \text { if } y \neq 0
\end{array}\right\}
$$

for $x, y \in U$.

For $x \in U$ let $\alpha(x): U \rightarrow U$ be the left translation $u \mapsto x+u$, and extend $\alpha(x)$ to $X$ by fixing the point $\infty$. Also extend $\tau$ to a map from $X$ to itself by $\tau(0)=\infty$ and $\tau(\infty)=0$. Define a family $\left(U_{x}\right)_{x \in X}$ of subgroups of the symmetric group of $X$ by

$$
U_{\infty}=\alpha(U), \quad U_{0}=\tau \circ U_{\infty} \circ \tau^{-1}, \quad U_{x}=\alpha(x) \circ U_{0} \circ \alpha(x)^{-1} \quad \text { for } x \in \dot{U} .
$$

This differs from the definition in [2] in that we write maps on the left of their arguments and accordingly let $U$ act on itself by left translations.

Now consider the case where $(U, \tau)$ defines a Moufang set $\mathbb{M}(U, \tau)$ (with underlying set $X)$ as in $[9,4.3]$. By [9, Theorem 4.4], this is the case if and only if $V=(U, U)$ as a division pair. In [9, Section 3], there is a construction of a Moufang set with underlying set $\mathbb{X}(V)$ for any division pair $V$, and then the map $\beta$ constructed above is in fact an isomorphism of Moufang sets $[9,4.3]$.

When comparing formulas in [2] with those in the present paper, care must be taken as to the order of the factors, because De Medts and Segev let their maps act on the right of $X$ whereas we let them act on the left. Also, one has to take 
into account that our maps $\alpha(x)$ are given by $\alpha(x) \cdot u=x+u$ whereas the maps $\alpha_{x}$ in [2] are defined by $u \alpha_{x}=u+x$. Consequently, the order of the factors in an identity has to be reversed, and the group law in $U$ has to be replaced by its opposite.

For $x \in \dot{U}$, the $\mu$-maps of [2] are

$$
\mu_{x}=\alpha_{(-x) \tau^{-1}}^{\tau} \alpha_{x} \alpha_{-\left(x \tau^{-1}\right)}^{\tau}
$$

As indicated above, this corresponds, in our setup, to the maps

$$
\mu(x)=\tau \circ \alpha\left(-\tau^{-1}(x)\right) \circ \tau^{-1} \circ \alpha(x) \circ \tau \circ \alpha\left(\tau^{-1}(-x)\right) \circ \tau^{-1},
$$

acting on $X$ on the left. (We use the subscripted notation $\alpha_{x}, \mu_{x}$ as in [2] and the notation with parentheses $\alpha(x), \mu(x)$ for our setup). By $[9,4.7]$, the maps $\mu(x)$ are related to the Weyl elements $\mathrm{w}_{+}(x)$ via $\beta$; more precisely, the diagram

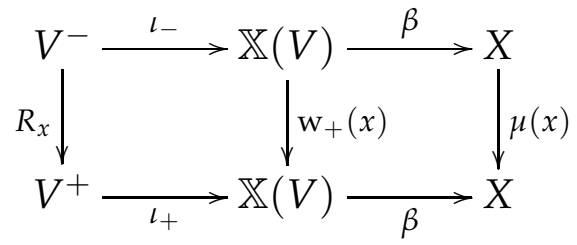

is commutative. In view of (4.8.2) and (4.8.3), and because $\dot{V}^{\sigma}=\dot{U}$, this implies the commutative diagram

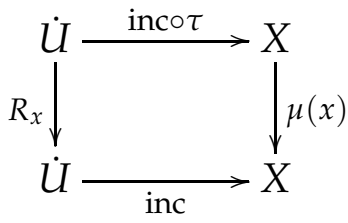

so we have the formula

$$
R_{x} y=\mu(x) \cdot \tau(y)=\mu(x) \cdot j_{-}(y)
$$

for $x, y \in \dot{U}$.

The first formula of $[2$, Theorem (1.1)] is

$$
\mu_{a \mu_{b}}=\mu_{b}^{-1} \mu_{a}^{-1} \mu_{b}
$$

where $a, b \in \dot{U}$. Let $x=b \in \dot{V}^{+}=\dot{U}$ and $y=\tau^{-1}(a) \in \dot{V}^{-}=\dot{U}$. Then by (4.8.5),

$$
a \mu_{b}=\mu(b) \cdot a=R_{b}\left(j_{+}(a)\right)=R_{b}\left(\tau^{-1}(a)\right) .
$$

Now (4.8.6) reads in our setting

$$
\mu\left(R_{b}\left(j_{+}(a)\right)\right)=\mu(b) \circ \mu(a)^{-1} \circ \mu(b)^{-1} .
$$

By the commutativity of the right hand square of (4.8.4), this is equivalent to the identity

$$
\mathrm{w}_{+}\left(R_{b} j_{+}(a)\right)=\mathrm{w}_{+}(b) \mathrm{w}_{+}(a)^{-1} \mathrm{w}_{+}(b)^{-1} .
$$


By (4.2.3), $\mathrm{w}_{+}(a)^{-1}=\mathrm{w}_{-}\left(j_{+}(a)\right)$, so this is a consequence of (4.3.1).

The second formula of $[2$, Theorem (1.1)] is

$$
\mu_{\left(a \tau^{-1}-b \tau^{-1}\right) \tau}=\mu_{-b} \mu_{b-a} \mu_{a}
$$

for $a, b \in \dot{U}$ with $a \neq b$. After reversing the order of the factors, and replacing the group structure of $U$ by its opposite, this becomes in our setup

$$
\mu\left(\tau\left(-\tau^{-1}(b)+\tau^{-1}(a)\right)\right)=\mu(a) \circ \mu(-a+b) \circ \mu(-b)
$$

which, again by (4.8.4), is equivalent to

$$
\mathrm{w}_{+}\left(j_{-}\left(-j_{+}(b)+j_{+}(a)\right)\right)=\mathrm{w}_{+}(a) \mathrm{w}_{+}(-a+b) \mathrm{w}_{+}(-b) .
$$

We claim that this is a consequence of (4.6.3):

$$
\mathrm{w}_{+}\left(x-{ }^{\vee} y\right) \mathrm{w}_{-}(y)=\mathrm{w}_{+}(x) \mathrm{w}_{-}\left(-x^{\vee}+y\right) .
$$

Indeed, put $x=-a$ and $y=j_{+}(b)$ in (4.8.9). Then $x-{ }^{\vee} y=-a+b$ and $-x^{\vee}+y=$ ${ }^{\vee}(-x)+y={ }^{\vee} a+j_{+}(b)=-j_{+}(a)+j_{+}(b)$. Hence (4.8.9) becomes

$$
\mathrm{w}_{+}(-a+b) \mathrm{w}_{-}\left(j_{+}(b)\right)=\mathrm{w}_{+}(-a) \mathrm{w}_{-}\left(-j_{+}(a)+j_{+}(b)\right) .
$$

By (4.2.3), $\mathrm{w}_{-}\left(j_{+}(b)\right)=\mathrm{w}_{+}(b)^{-1}=\mathrm{w}_{+}(-b)$ and $\mathrm{w}_{+}(-a)=\mathrm{w}_{+}(a)^{-1}$. Moreover, putting $v=-j_{+}(a)+j_{+}(b)$, we have $\mathrm{w}_{-}(v)=\mathrm{w}_{+}\left(v^{\vee}\right)($ by $(4.2 .2))=$ $\mathrm{w}_{+}\left(j_{-}(-v)\right)=\mathrm{w}_{+}\left(j_{-}\left(-j_{+}(b)+j_{+}(a)\right)\right)$. Substituting this in (4.8.10) and rearranging factors yields (4.8.8).

4.9 The Bergmann operators. For quasi-invertible $(x, y) \in V^{\sigma} \times V^{-\sigma}$ let $B(x, y): V^{\sigma} \rightarrow$ $V^{\sigma}$ be defined by

$$
B(x, y)=\left\{\begin{array}{ll}
R\left(x-{ }^{\vee} y\right) R_{y} & \text { if } y \neq 0 \\
\operatorname{Id}_{V^{\sigma}} & \text { if } y=0
\end{array}\right\} .
$$

In case $V$ is a Jordan division pair, these are the so-called Bergmann operators. Clearly, $B(x, y)$ is an automorphism of the group $V^{\sigma}$, and $B(0, y)=\operatorname{Id}_{V^{\sigma}}$ by (4.2.5). We now prove a number of identities for the $B(x, y)$ which generalize identities from [5].

4.10 Lemma. Let $V$ be a division pair and let $(x, y) \in \dot{V}^{\sigma} \times \dot{V}^{-\sigma}$ be quasi-invertible. Then the following formulas hold:

$$
\begin{aligned}
B(x, y) & =R_{x} R\left(-x^{\vee}+y\right), \\
R_{x} & =B(x, y) R\left(x^{y}\right), \\
R_{-x} & =R\left(-\left(x^{y}\right)\right) B(-y,-x), \\
B(x, y) R_{-x} & =R\left(-{ }^{\vee} x+R_{x} y\right), \\
R_{-y} B(x, y) & =R\left(R_{-y} x+{ }^{\vee \vee}(-y)\right) .
\end{aligned}
$$

Proof. Let $\mathscr{G}$ and $V \cong \Delta(\mathscr{G})$ as in 4.1 . Then $B(x, y)$ is related to $\mathrm{b}_{\sigma}(x, y)$ by

$$
\operatorname{Int}\left(\mathrm{b}_{\sigma}(x, y)\right) \mathrm{e}_{\sigma}(z)=\mathrm{e}_{\sigma}(B(x, y) z)
$$




$$
\operatorname{Int}\left(\mathrm{b}_{\sigma}(x, y)\right) \mathrm{e}_{-\sigma}(v)=\mathrm{e}_{-\sigma}\left(B(-y,-x)^{-1} v\right) .
$$

Indeed, (4.10.6) follows from the first equality of (4.6.3) and (4.1.6), and (4.10.7) is a consequence of (4.6.4). Now (4.10.2) is immediate from the second equality of (4.6.3) and again (4.1.6). The remaining formulas follow in the same way from the corresponding ones in Corollary 4.7.

For quasi-invertible $(x, y) \in V^{+} \times V^{-}$, we put

$$
\beta(x, y)=\left(B(x, y), B(-y,-x)^{-1}\right): V \rightarrow V .
$$

Then $\beta(x, y)$ is, by Proposition 4.3(b), the composition of two isomorphisms:

$$
\beta(x, y)=\left(R_{x}, R\left(x^{\vee}\right)\right) \circ\left(R\left(-x^{\vee}+y\right), R\left(\left(-x^{\vee}+y\right)^{\vee}\right)\right): V \rightarrow V^{\text {op }} \rightarrow V,
$$

and hence an automorphism of the division pair $V$.

The following corollaries of Proposition 4.6 are the analogues of similar results for Jordan pairs [5, §3].

4.11 Corollary. Let $(x, y) \in V^{\sigma} \times V^{-\sigma}$ be quasi-invertible, let $t \in V^{-\sigma}$ and $z \in V^{\sigma}$.

(a) $(x, y+t)$ is quasi-invertible if and only if $\left(x^{y}, t\right)$ is quasi-invertible, and then

$$
\begin{aligned}
x(y+t) & ={ }^{x} y+B(-y,-x)^{-1}\left({ }^{\left(x^{y}\right)} t\right), \\
\mathbf{b}_{\sigma}(x, y+t) & =\mathbf{b}_{\sigma}(x, y) \mathbf{b}_{\sigma}\left(x^{y}, t\right), \\
x^{y+t} & =\left(x^{y}\right)^{t} .
\end{aligned}
$$

(b) $(z+x, y)$ is quasi-invertible if and only if $(z, x y)$ is quasi-invertible, and in this case,

$$
\begin{aligned}
(z+x) y & ={ }^{z}\left({ }^{x} y\right) \\
\mathrm{b}_{\sigma}(z+x, y) & =\mathrm{b}_{\sigma}\left(z{ }^{x} y\right) \mathrm{b}_{\sigma}(x, y), \\
(z+x)^{y} & =B(x, y)^{-1}\left(z^{(x y)}\right)+x^{y} .
\end{aligned}
$$

Proof. Since $(x, y)$ is quasi-invertible, we have by Proposition 4.6,

$$
\begin{aligned}
\mathbf{e}_{\sigma}(x) \mathrm{e}_{-\sigma}(y+t) & =\mathbf{e}_{\sigma}(x) \mathrm{e}_{-\sigma}(y) \mathrm{e}_{-\sigma}(t) \\
& =\mathrm{e}_{-\sigma}\left({ }^{x} y\right) \mathrm{b}_{\sigma}(x, y) \mathbf{e}_{\sigma}\left(x^{y}\right) \mathrm{e}_{-\sigma}(t),
\end{aligned}
$$

and this belongs to $\Omega^{\sigma}$ if and only if $\mathrm{e}_{\sigma}\left(x^{y}\right) \mathrm{e}_{-\sigma}(t) \in \Omega^{\sigma}$, that is, $\left(x^{y}, t\right)$ is quasiinvertible. Assume this to be the case. Then using (4.6.2) on the left and right hand side of the above equation yields, by (4.10.7),

$$
\begin{aligned}
\mathrm{e}_{\sigma}(x) \mathrm{e}_{-\sigma}(y+t) & =\mathrm{e}_{-\sigma}\left({ }^{x}(y+t)\right) \mathrm{b}_{\sigma}(x, y+t) \mathrm{e}_{\sigma}\left(x^{y+t}\right) \\
& =\mathrm{e}_{-\sigma}\left({ }^{x} y\right) \mathrm{b}_{\sigma}(x, y) \mathrm{e}_{-\sigma}\left({ }^{\left(x^{y}\right)} t\right) \mathrm{b}_{\sigma}\left(x^{y}, t\right) \mathrm{e}_{\sigma}\left(\left(x^{y}\right)^{t}\right) \\
& \left.=\mathrm{e}_{-\sigma}\left({ }^{x} y+B(-y,-x)^{-1}\left({ }^{y}\right) t\right)\right) \mathbf{b}_{\sigma}(x, y) \mathbf{b}_{\sigma}\left(x^{y}, t\right) \mathbf{e}_{\sigma}\left(\left(x^{y}\right)^{t}\right) .
\end{aligned}
$$

By Lemma 2.3(e), we conclude that the components in $U^{-\sigma}, H$ and $U^{\sigma}$ of both sides agree. This proves (4.11.1) - (4.11.3). The proof of $(b)$ is analogous. 
4.12 Corollary. Let $(x, y) \in \dot{V}^{\sigma} \times \dot{V}^{-\sigma}$ be quasi-invertible. Then

$$
\begin{aligned}
& \left(x^{y}\right)^{\vee \vee}=x^{\vee \vee}+R_{-x}\left({ }^{x} y\right), \\
& { }^{\vee \vee}\left({ }^{x} y\right)=R_{y}\left(x^{y}\right)+{ }^{\vee \vee} y, \\
& B(x, y) x^{y}=\left[-R_{x} y+{ }^{\vee \vee} x\right]^{\vee \vee} \text {. }
\end{aligned}
$$

Proof. Put $z:=B(x, y) x^{y} \in V^{\sigma}$ and $v=-y+x^{\vee} \in V^{-\sigma}$. From (4.6.2), (4.6.3) and (4.10.6) we obtain

$$
\begin{aligned}
\mathrm{e}_{\sigma}(x) \mathrm{e}_{-\sigma}(y) & =\mathrm{e}_{-\sigma}\left({ }^{x} y\right) \mathrm{b}_{\sigma}(x, y) \mathrm{e}_{\sigma}\left(x^{y}\right)=\mathrm{e}_{-\sigma}\left({ }^{x} y\right) \mathrm{e}_{\sigma}(z) \mathrm{b}_{\sigma}(x, y) \\
& =\mathrm{e}_{-\sigma}\left({ }^{x} y\right) \mathrm{e}_{\sigma}(z) \mathrm{w}_{\sigma}(x) \mathrm{w}_{-\sigma}(-v) .
\end{aligned}
$$

Then, using (4.2.2) and (4.2.3) as well as (4.2.4) and (4.1.6),

$$
\begin{aligned}
\mathrm{e}_{-\sigma}\left({ }^{x} y\right) \mathrm{e}_{\sigma}(z) & =\mathrm{e}_{\sigma}(x) \mathrm{e}_{-\sigma}(y) \mathrm{w}_{-\sigma}(v) \mathrm{w}_{\sigma}(x)^{-1} \\
& =\mathrm{e}_{\sigma}(x) \mathrm{e}_{-\sigma}(y) \mathrm{e}_{-\sigma}(v) \mathrm{e}_{\sigma}\left(v^{\vee}\right) \mathrm{e}_{-\sigma}\left(v^{\vee \vee}\right) \mathrm{w}_{\sigma}(x)^{-1} \\
& =\mathrm{e}_{\sigma}(x) \mathrm{e}_{-\sigma}(y+v) \mathrm{e}_{\sigma}\left(v^{\vee}\right) \mathrm{e}_{-\sigma}\left(v^{\vee \vee}\right) \mathrm{w}_{\sigma}(x)^{-1} \\
& =\mathrm{e}_{\sigma}(x) \mathrm{e}_{-\sigma}\left(x^{\vee}\right) \mathrm{e}_{\sigma}\left(v^{\vee}\right) \mathrm{e}_{-\sigma}\left(v^{\vee \vee}\right) \mathrm{w}_{\sigma}(x)^{-1} \\
& =\mathrm{w}_{\sigma}(x) \mathrm{e}_{\sigma}\left(-x^{\vee \vee}+v^{\vee}\right) \mathrm{e}_{-\sigma}\left(v^{\vee \vee}\right) \mathrm{w}_{\sigma}(x)^{-1} \\
& =\mathrm{e}_{-\sigma}\left(R\left(x^{\vee}\right)\left[-x^{\vee \vee}+v^{\vee}\right]\right) \mathrm{e}_{\sigma}\left(R_{x}\left(v^{\vee \vee}\right)\right) \in U^{-\sigma} U^{\sigma} .
\end{aligned}
$$

Here the first factor on the left and on the right belongs to $U^{-\sigma}$ while the second factors belong to $U^{\sigma}$. Since $U^{\sigma} \cap U^{-\sigma}=\{1\}$ we conclude

$$
\begin{aligned}
x y & =R\left(x^{\vee}\right)\left[-x^{\vee \vee}+v^{\vee}\right], \\
B(x, y) x^{y} & =R_{x}\left(v^{\vee \vee}\right) .
\end{aligned}
$$

By (4.2.6), $R\left(x^{\vee}\right)^{-1}=R_{-x}$. Hence (4.12.4) implies

$$
R_{-x}(x y)=-x^{\vee \vee}+v^{\vee}=-x^{\vee \vee}+\left(-y+x^{\vee}\right)^{\vee}=-x^{\vee \vee}+\left(x^{y}\right)^{\vee \vee}
$$

which is equivalent to (4.12.1). We obtain (4.12.2) from (4.12.1) by the substitution $x \mapsto-y, y \mapsto-x$ and by taking the negative of both sides. Finally, by (4.3.5), $R_{x}\left(v^{\vee \vee}\right)=\left(R_{x} v\right)^{\vee \vee}$, and $R_{x} v=R_{x}\left(-y+x^{\vee}\right)=-R_{x} y+{ }^{\vee} x$ (by (4.2.1)), so (4.12.3) follows from (4.12.5).

4.13 Special rank one groups and division pairs. Following Timmesfeld [12], a rank one group is called special if the $j$-maps of Lemma 2.2 commute with group inversion. This is a property of the associated division pair: in the setting of $4.1, \mathscr{G}$ is special if and only if $V$ is special in the sense that, for all $x \in \dot{V}^{\sigma}$, ${ }^{\vee} x=x^{\vee}$. The reader should be warned that this has nothing to do with the notion of speciality for Jordan pairs (embeddability in an associative system).

In a special division pair, we define

$$
x^{-1}={ }^{\vee} x=x^{\vee}
$$


for $x \in \dot{V}^{\sigma}$, so that

$$
j(x)=-x^{-1} .
$$

Beware that $x^{-1}$ is not the inverse of $x$ in the group $V^{\sigma}$, which is $-x$, but rather belongs to $\dot{V}^{-\sigma}$. This notation is consistent with the one for the inverse in Jordan pairs $[9,1.8]$.

In a special division pair, we have ${ }^{\vee \vee} x=x=x^{\vee \vee}$, so the formulas of 4.9 and Corollary 4.12 simplify. Formula (4.2.1) now reads

$$
x=R_{x} x^{-1}=R_{-x} x^{-1}
$$

and hence the $j$-maps can be reconstructed from the $R$-operators by

$$
j(x)=-R_{x}^{-1} x .
$$

We also have

$$
(x, y) \text { quasi-invertible } \Longleftrightarrow \quad(y, x) \text { quasi-invertible, }
$$

as follows easily from the definition in 4.4 .

4.14 Lemma. The following conditions are equivalent:

(i) $\mathscr{G}=\left(G, U^{+}, U^{-}\right)$is special and the $U^{ \pm}$are abelian,

(ii) $V$ is special and the groups $V^{\sigma}$ are abelian,

(iii) the group inversion maps $i: V^{\sigma} \rightarrow V^{\sigma}$ define an automorphism $(i, i): V \rightarrow$ $V$ of the division pair $V$.

In this case,

$$
\begin{gathered}
R_{-x}=R_{x}, \\
\mathrm{w}_{\sigma}(x)^{2} \in \mathscr{Z}(G),
\end{gathered}
$$

for all $x \in \dot{V}^{\sigma}$. Moreover, for quasi-invertible $(x, y) \in \dot{V}^{\sigma} \times V^{-\sigma}$ the following formulas hold:

$$
\begin{aligned}
B(x, y) & =B(-x,-y), \\
y_{x} & =\left(x^{-1}-y\right)^{-1}=x^{y}, \\
x^{y} & =x+R_{x}\left(y^{x}\right), \\
B(x, y) x^{y} & =x-R_{x} y, \\
B(x, y) R_{x} & =R_{x} B(y, x)=R\left(x-R_{x} y\right) .
\end{aligned}
$$

Remark. (4.14.1) was proved in [2, Lemma 5.1].

Proof. (i) $\Longleftrightarrow$ (ii) is evident from the definitions, and (ii) $\Longleftrightarrow$ (iii) holds because $i: V^{\sigma} \rightarrow V^{\sigma}$ is a group automorphism if and only if $V^{\sigma}$ is abelian. Assume these conditions hold. Then $i\left(R_{x} y\right)=R_{i(x)} i(y)$ by (1.1.2). But $R_{x}$ is a group homomorphism, hence commutes with $i$, so we have $i\left(R_{x} y\right)=R_{x} i(y)=R_{i(x)} i(y)$ for all $y \in V^{\sigma}$, which proves (4.14.1). Similarly, (4.14.3) is a consequence of (iii). 
To prove (4.14.2), let $y \in V^{-\sigma}$. Then by (4.1.6), (4.14.1) and (4.2.3),

$$
\begin{aligned}
\operatorname{Int}\left(\mathrm{w}_{\sigma}(x)\right) \mathrm{e}_{-\sigma}(y) & =\mathrm{e}_{\sigma}\left(R_{x} y\right)=\mathrm{e}_{\sigma}\left(R_{-x} y\right)=\operatorname{Int}\left(\mathrm{w}_{\sigma}(-x)\right) \mathrm{e}_{-\sigma}(y) \\
& =\operatorname{Int}\left(\mathrm{w}_{\sigma}(x)^{-1}\right) \mathrm{e}_{-\sigma}(y) .
\end{aligned}
$$

This shows $\left(\left(\mathrm{w}_{\sigma}(x)^{2}, U^{-\sigma}\right)\right)=\{1\}$. But $\mathrm{w}_{\sigma}(x)=\mathrm{w}_{-\sigma}\left(x^{\vee}\right)$ by (4.2.2), so the same argument shows that $\left(\left(\mathrm{w}_{\sigma}(x)^{2}, U^{\sigma}\right)\right)=\{1\}$. This implies (4.14.2).

If $(x, y)$ is quasi-invertible then by (4.4.1) and (4.4.2),

$$
{ }^{y} x=\left({ }^{\vee} x-y\right)^{\vee}={ }^{\vee}\left(-y+x^{\vee}\right)=x^{y}
$$

since $V^{-\sigma}$ is abelian. Formula (4.14.5) follows from (4.12.1), (4.14.1) and (4.14.4), and (4.14.6) follows from (4.12.3) as well. Finally, (4.14.7) is a consequence of (4.14.1), (4.10.4) and (4.10.5).

\section{References}

[1] P. Abramenko and K. S. Brown, Buildings, Graduate Texts in Mathematics, vol. 248, Springer, New York, 2008.

[2] T. De Medts and Y. Segev, Identities in Moufang sets, Trans. Amer. Math. Soc. 360 (2008), no. 11, 5831-5852.

[3] T. De Medts and R. Weiss, Moufang sets and Jordan division algebras, Math. Ann. 335 (2006), no. 2, 415-433.

[4] J. R. Faulkner, Stable range and linear groups for alternative rings, Geom. Dedicata 14 (1983), 177-188.

[5] O. Loos, Jordan pairs, Springer-Verlag, Berlin, 1975, Lecture Notes in Mathematics, Vol. 460.

[6] _ On algebraic groups defined by Jordan pairs, Nagoya Math. J. 74 (1979), 23-66.

[7] _ Elementary groups and stability for Jordan pairs, K-Theory 9 (1995), 77-116.

[8] _ Steinberg groups and simplicity of elementary groups defined by Jordan pairs, J. Algebra 186 (1996), no. 1, 207-234.

[9] _ Division pairs: a new approach to Moufang sets, to appear in Adv. Geom. Preprint at http://molle.fernuni-hagen.de/ loos/jordan/ archive/divmou/divmou.pdf, 2012.

[10] O. Loos and E. Neher, Steinberg groups defined by Jordan pairs, in preparation.

[11] R. Steinberg, Lectures on Chevalley groups, Yale University Lecture Notes, New Haven, Conn., 1967. 
[12] F. G. Timmesfeld, Abstract root subgroups and simple groups of Lie type, Monographs in Mathematics, vol. 95, Birkhäuser Verlag, Basel, 2001.

[13] _ Quadratic rank-one groups and quadratic Jordan division algebras, Proc. London Math. Soc. 95 (2007), no. 3, 156-178.

[14] _ Algebraic structures related to universal covers of special rank one groups, J. Algebra 322 (2009), no. 1, 13-20.

[15] J. Tits, Twin buildings and groups of Kac-Moody type, Groups, combinatorics \& geometry (Durham, 1990), London Math. Soc. Lecture Note Ser., vol. 165, Cambridge Univ. Press, Cambridge, 1992, pp. 249-286.

Fakultät für Mathematik und Informatik, FernUniversität in Hagen, 58097 Hagen, Germany email:Ottmar.Loos@FernUni-Hagen.de 